Florida International University

FIU Digital Commons

6-19-2019

\title{
Multiple Criteria Decision Models for Nurse-Patient Assignment: Balancing Workload and Continuity of Care
}

Haoqiang Jiang

hjian006@fiu.edu

Follow this and additional works at: https://digitalcommons.fiu.edu/etd

Part of the Management Information Systems Commons

\section{Recommended Citation}

Jiang, Haoqiang, "Multiple Criteria Decision Models for Nurse-Patient Assignment: Balancing Workload and Continuity of Care" (2019). FIU Electronic Theses and Dissertations. 4248.

https://digitalcommons.fiu.edu/etd/4248

This work is brought to you for free and open access by the University Graduate School at FIU Digital Commons. It has been accepted for inclusion in FIU Electronic Theses and Dissertations by an authorized administrator of FIU Digital Commons. For more information, please contact dcc@fiu.edu. 


\title{
FLORIDA INTERNATIONAL UNIVERSITY
}

\author{
Miami, Florida
}

MULTIPLE CRITERIA DECISION MODELS FOR NURSE-PATIENT ASSIGNMENT: BALANCING WORKLOAD AND CONTINUITY OF CARE

A dissertation submitted in partial fulfillment of

the requirements of the degree of DOCTOR OF PHILOSOPHY

in

BUSINESS ADMINISTRATION

by

Haoqiang Jiang 
To: Dean Joanne Li

College of Business

This dissertation, written by Haoqiang Jiang, and entitled by Multiple Criteria Decision Models for Nurse-patient Assignment: Balancing Workload and Continuity of Care, having been approved in respect to style and intellectual content, is referred to you for judgement.

We have read this dissertation and recommend that it be approved.

George M. Marakas

Paulo Gomes

\section{Ellen Brown}

Hemang Subramanian

Debra VanderMeer, Major Professor

Date of Defense: June 19, 2019

This dissertation of Haoqiang Jiang is approved.

Dean Joanne Li
College of Business

Andres G.Gil

Vice President for Research and Economic Development and Dean of the University Graduate School

Florida International University, 2019 
(C) Copyright 2019 by Haoqiang Jiang

All rights reserved. 


\section{ACKNOWLEDGMENTS}

I want to thank my mother and my wife. Without family support, I could not succeed in these five years.

I want to express my most appreciation to my committee chair, Dr. Debra VanderMeer, who had guided me on how to think like a scholar and devoted lots of time and thinking to this research project. Without her guidance, I could not finish this dissertation on time.

I am deeply indebted to my committee member Dr. Paulo Gomes, who guided me to explore more relevant literature and build evaluation methods to verify the research results. I want to extend my sincere thanks to my committee members, Dr. George Marakas, Dr. Ellen Brown, and Dr. Hemang Subramanian, who provided me lots of useful comments and suggestions for this research project.

Last but not least, I want to thank the Department of Information Systems and Business Analytics, College of Business, and FIU to provided me an excellent opportunity to study and grow up. 


\begin{abstract}
OF THE DISSERTATION
MULTIPLE CRITERIA DECISION MODELS FOR NURSE-PATIENT

ASSIGNMENT: BALANCING WORKLOAD AND CONTINUITY OF CARE

by

Haoqiang Jiang
\end{abstract}

Florida International University, 2019

Miami. Florida

Professor Debra VanderMeer, Major Professor

Continuity of care is critical for delivering high-quality care, yet has seldom been considered in models supporting nurse-patient assignment decisions within inpatient units. Research in the nursing literature suggests that assigning nurses to patients they have cared for previously can help reduce care-related error rates, and increase patient satisfaction. However, it is also essential to ensure that patient workloads are allocated to nursing staff in a balanced manner to avoid overwork and burnout.

This study investigates the tradeoffs associated with the assignment of patients to nurses in inpatient settings under the dual objective of maximizing continuity of care and minimizing workload imbalance. We develop a hybrid method that balances the need for fair workload distribution and continuity of care, and demonstrate the extent of the tradeoff between the level of continuity achieved and the associated cost in workload balance. To reduce the impact of this tradeoff, we relax the goal of maximizing continuity by introducing an acuity threshold. Here, patients with acuity values above the threshold 
are targeted for continuity-based assignment, and remaining patients are assigned to minimize workload imbalance.

We evaluate the utility of introducing the threshold under a variety of hospital environmental conditions using a simulation model of the inpatient environment. Our findings show that it is possible to provide a substantial continuity assignment with a marginal impact on workload imbalance under the hybrid policy using the acuity threshold. In virtually all cases studied, the results show that it is possible to use the acuity threshold and gain the benefits of continuity of care, even under conditions of a strong preference for minimizing acuity imbalance. 


\section{TABLE OF CONTENTS}

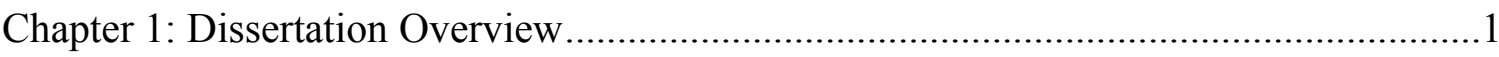

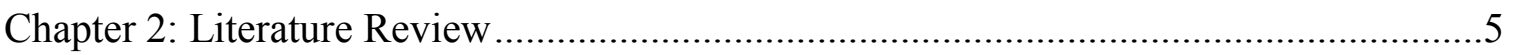

Work Assignment in Operational Management ……………......................................

Work Assignment in NPA Problem ........................................................................

Acuity and Workload Balance in NPA ……………..............................................

Subjective Workload and Role of Task Familiarity ……….......................................10

Continuity of Care in NPA ………………………............................................12

Chapter 3: Problem Description and Research Method.................................................14

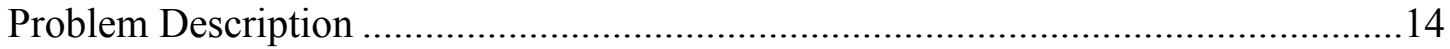

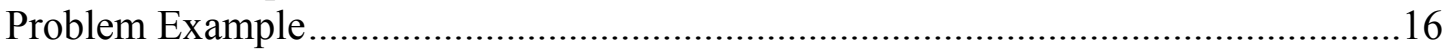

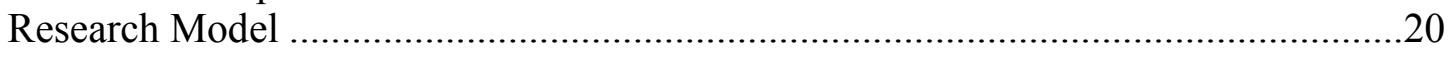

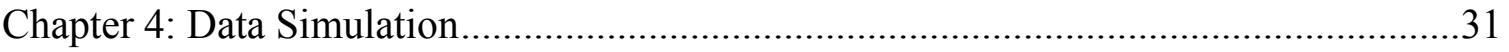

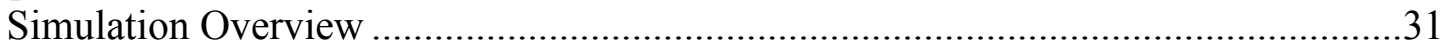

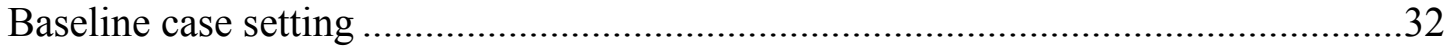

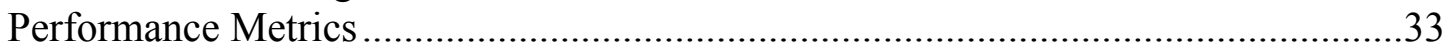

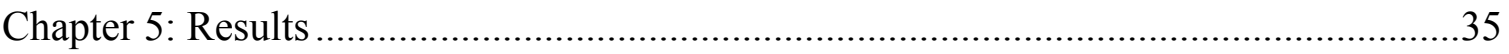

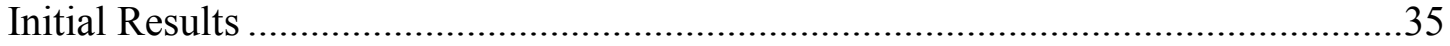

Acuity threshold policy based on initial results ..........................................................39

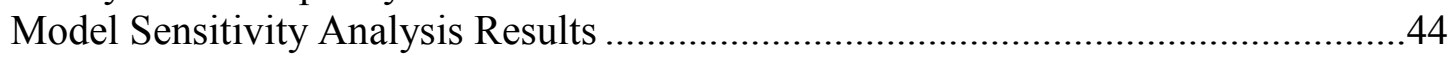

Chapter 6: Discussion, Conclusions, and Recommendations .............................................67

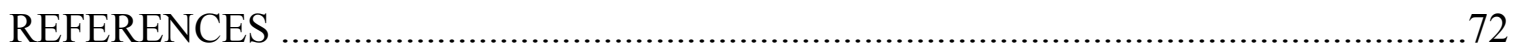

VITA …… 


\section{LIST OF TABLES}

TABLE

PAGE

Chapter 3:

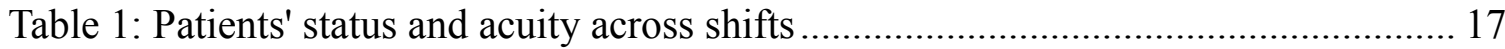

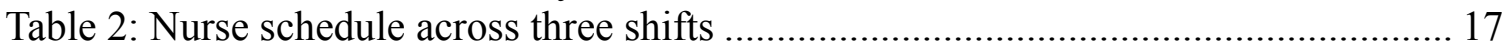

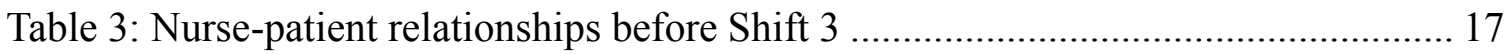

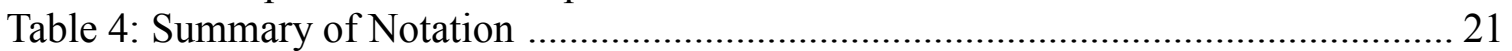

Chapter 5:

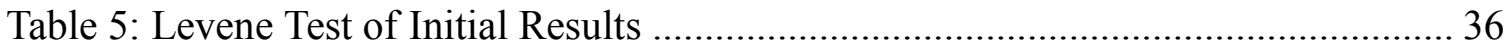

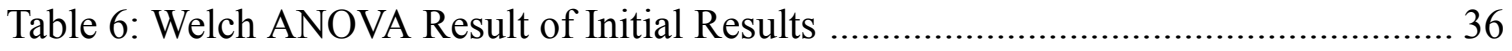

Table 7: AAI and PCC Performance for Simple Heuristic Methods ................................ 37

Table 8: AAI and PCC Performance for Acuity Threshold Policy ................................ 41

Table 9: Preference Model for Baseline Case .................................................................... 42

Table 10: Summary of 5 Different Environment Cases ................................................... 45

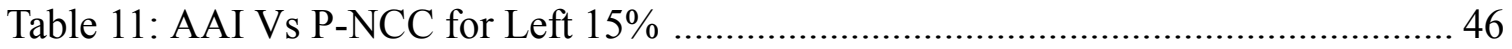

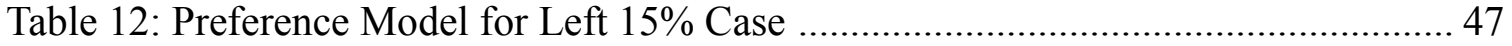

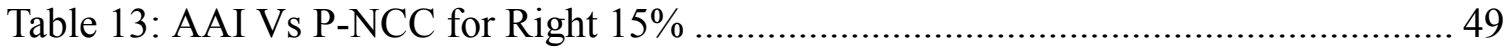

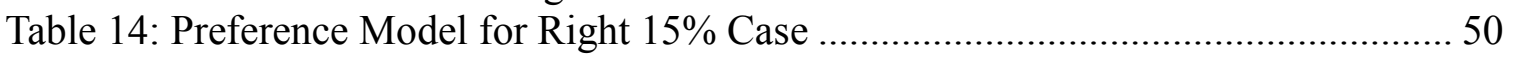

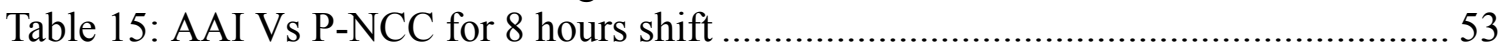

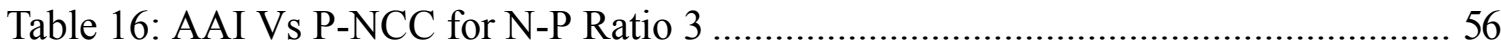

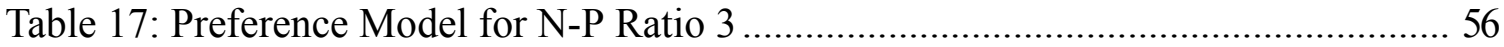

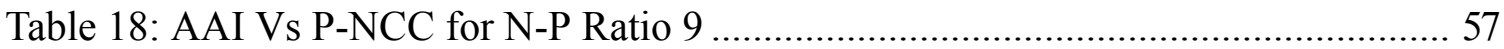

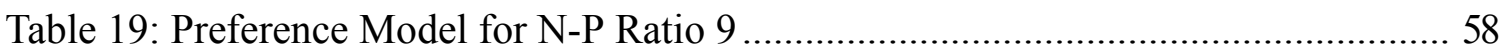

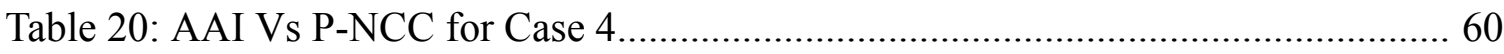

Table 21: Preference Model for 20\% Special Requirement Case.................................. 61

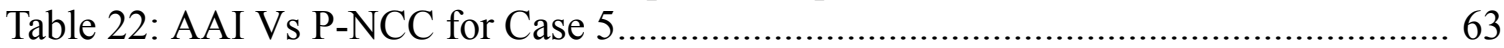

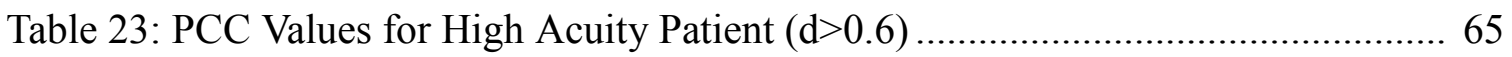




\section{LIST OF FIGURES}

FIGURE PAGE

Chapter 3:

Figure 1: Two different NPA policies for the same patient census ................................19

\section{Chapter 5:}

Figure 2: AAI Vs P-NCC for Baseline Case ..................................................................42

Figure 3: Best Acuity Threshold based on Preference Weight ........................................43

Figure 4: [A] High Weight Area for P-NCC; [B] Example Horizontal Run .....................44

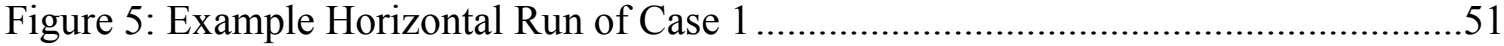

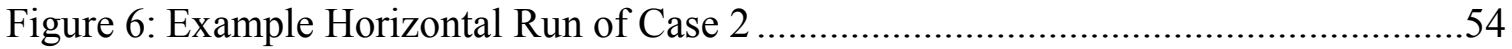

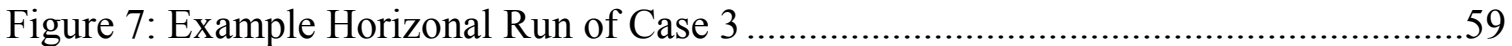

Figure 8: Example Horizontal Run of Case 4 ............................................................61

Figure 9: Example Horizontal Run of Case 5 ...........................................................64 


\section{Chapter 1: Dissertation Overview}

With the advent of value-based healthcare, it is important for hospitals to rethink their operational systems designing for both efficiency and service. Continuity of care has received little attention as an organizing element in hospital inpatient care settings; yet, building structures that foster continuity of care is an important element in a patientcentered culture that aims to achieve higher patient satisfaction. Continuity of care also contributes to the satisfaction of clinicians (Bowers et al., 2015), and to patient outcomes through consistency of care and reduction of handovers.

Nurse-patient assignment is an important daily routine in hospitals, as it ensures the distribution of patient care workload among available nurses (Liang and Turkcan, 2015). Finding an appropriate nurse for patients is a pressing issue for nursing managers, who need to reasonably arrange patients for each nurse considering nurse skills, patient care needs (Aiken et al., 2014) and workload difference among distinct nurses (Baker et al., 2010). Previous studies of nurse-patient assignment (NPA) focus on quantitative factors like number of patients, location of patients, and patient acuity to balance the workload among nurses (Mullinax and Lawley, 2002, Schaus et al., 2009); recent studies suggest that qualitative factors such as continuity of care are also important to charge nurses in nurse workload assignment decisions (Massey et al., 2017, Plover, 2017). While continuity of care (CC) has been considered as critical criteria in nurse assignment models of home health care, where the nurse assignment decisions span multiple periods (Fikar and Hirsch, 2017), it is underexplored in NPA problems in inpatient settings. A key concern is that continuity of care assignment collides with the emphasis on operational efficiency 
(Lanzarone and Matta, 2014). Using continuity of care as a factor in assigning nurses to patients can come at a cost in workload balance, i.e., increasing the level of continuity in assignments may lead to increased workload imbalance. Perceived lack of balance in workloads can lead to higher levels of nurse dissatisfaction (Allen, 2015), which can affect patient care quality as well as nurses' stress levels and rates of burnout. The questions we ponder are: what is the nature of the tradeoff between workload balance and continuity of care? What mechanisms can be used to break that tradeoff?

To address the issues above, we reexamine the nurse-patient assignment problem and consider the impact of continuity of care on assignment decisions and workload distribution. NPA under continuity of care (CC) consists of assigning each patient to a nurse they have been assigned in a previous shift during the current hospital stay, chosen among the compatible ones, in order to minimize the number of staff handovers during a patient length of stay. The amount of work required by the patient during one shift is estimated by patient acuity and is the basis for determining workload balance. This problem can be formulated as a bi-criteria integer-programming problem whose objectives are maximizing workload balance and continuity of care. In addition, the problem features several constraints: the number of patients assigned to a nurse cannot exceed the nursepatient ratio (refer to N-P Ratio); nurses cannot work on consecutive shifts; patient needs require nurses with specific skills. We create a simulation model that reflects the environment of a typical general medical-surgical unit, with a set of nurses scheduled to cover shifts, and a set of patients admitted to and discharged from the unit, and then incorporated into the discrete event simulation environment different NPA policies in order 
to understand the performance of each policy and explore the tradeoffs between workload balance and continuity of care. We develop a hybrid NPA policy to continue to balance workloads using acuity as a quantitative measure, while introducing continuity of care to allow nurses to care for the same patients across multiple shifts. We compare the performance of the hybrid policy with control policies, namely a workload balancing policy (WB), a numeric balancing policy that distributes patient census (NB), and a pure CC policy. Each of these policies ensures numeric balance.

The simulation results show a clear tradeoff between continuity of care and balanced workload distribution. To alleviate such tradeoff, we consider a refinement of the hybrid method, the use of an acuity threshold to segment the patient pool, relaxing the goal of maximizing continuity of care assignment for all patients. We borrow the idea which suggests that in order to break the tradeoff between efficiency and service firms should target customers on the basis of variability type (Frei, 2006). The acuity threshold targets higher-acuity patients for continuity of care assignment. These patients need more care than low-acuity patients (Stanton and Stanton, 2004) and may also be the ones that further benefit from familiarity with the caregiver. Results show that we can achieve up to a 198.70 percentage-point increase in CC assignments, for 137.75 costs in terms of workload balance over a policy focused solely on workload balancing. To test the robustness of results we further investigate whether the proposed acuity threshold results hold under different simulation settings. We consider simulation scenarios where we vary the distribution of patient's acuity level, nursing shift length, the nurse-to-patient ratio and the proportion of patients with special care needs. 
This research makes several contributions to the literature and managerial decisionmaking. An important finding of this study is that emphasizing continuity-of-care assignments for the highest acuity patients while emphasizing workload balance assignments for the lower acuity patients seems to enable significant increases in the continuity of care with reduced impact on workload balance. The simplicity of the proposed method facilitates its implementation in practice. Most academic nurse scheduling work has not been incorporated into practice (Kellogg and Walczak, 2007). Recent work has shown success in implementation when the effort required to develop an assignment solution is reduced (Cohn et al., 2009).

The remainder of the article is organized as follows. In the next section, we provide a review of the related literature. Then, we discuss different policies and introduce our method. We describe a set of simulation experiments for the nurse-patient assignment. Finally, we present a discussion of the results and limitations of our work, and describe opportunities for future work. 


\section{Chapter 2: Literature Review}

\section{Work Assignment in Operational Management}

Workload assignment is a routine job for managers in an organization. Managers seek to divide workload in a fair and balanced manner to the employees, workload assignments should be suitable for the employees' capability, should meet both the organization's and the employees' schedule needs, and should maximize the efficiency of operations. Furthermore, the assignment should follow guidelines of the organizational setting or regulatory framework and be flexible in response to urgent requirements in real time. A good workload assignment could benefit organizational performance, working efficiency, and employee satisfaction (Loucks and Jacobs, 1991).

There is a vast body of research on task scheduling and personnel scheduling (Van den Bergh et al., 2013), scheduling hospital personnel, in particular, is recognized as a complex problem (Burke et al., 2004). Such studies typically present optimization models based on quantitative factors in order to improve task scheduling and rotation among employees in organizations (Burke et al., 2004), and workload distribution among people in a working unit (Sun et al., 2014). These studies contribute to the literature by proposing methods to solve the problems of balancing assignments or optimizing the standard working process and working scheduling among people or production lines. Scheduling methods seek to optimize for quantitative factors like production rate, resource utilization (e.g., process time, inventory, storage space, and length of duties), cost of production, and task schedule time (Hillier, 2013, Abdelghany et al., 2008). 
The complexity of workload distribution problems is partly determined by the need to consider the requirements of different stakeholders. More attention is being devoted to satisfying employee needs in staffing and scheduling decisions (Van den Bergh et al., 2013). For instance, studies of airline scheduling and crew assignment attempt to balance the desired outcomes from managers, flight crews, ground crews, and passengers against the organization's production and schedule. Multiple quantitative factors are considered associated with stakeholder goals, including the scheduled block-time of airplanes, minimum crew rest after duty, crew connections between scheduled flights, passenger connections, and the duration of scheduled maintenance after a flight leg. (Lee et al., 2007, Lee et al., 2008, Burke et al., 2010). Similarly, studies of hospital personnel scheduling have considered physicians preferences while balancing workload distribution (Fügener et al., 2015).

\section{Work Assignment in NPA problem}

The nurse-patient assignment (NPA) literature has grown out of a vast literature on staff scheduling in healthcare organizations. In staff tour scheduling, organizations operate seven days a week, with more than one shifts a day (such as hospitals) and the particular tour (i.e., hours of the day and days of the week) in which the employee must work is specified. Various performance criteria have been used in the scheduling literature. The recent study classifies these criteria as patient-related, staff-related and management related measures (Xiang, 2017). Patient-related criteria include response or wait time, length of stay, and patient deferral. Staff-related criteria include overtime, workload and scheduling preferences. Management-related criteria include resource utilization and resource leveling 
and cost. Additional patient requirements include patient priority or need for specialized nursing skills (Liang and Turkcan, 2015, Vile et al., 2017) and continuity of care (Bowers et al., 2015). More attention is being devoted to satisfying employee needs in staffing and scheduling decisions (Van den Bergh et al., 2013). For instance, studies of hospital personnel scheduling have considered physicians preferences while balancing workload distribution (Fügener et al., 2015).

NPA follows nurse-scheduling decisions and is typically made at the beginning of each shift. The assignment problem involves matching patients to nursing staff in such a way that performance objectives are optimized. The complexity of NPA problems is partly determined by the need to consider the requirements of different stakeholders.

We find in the literature different descriptions and models for NPA based on the distinct settings in which the NPA problem has been investigated: outpatient, inpatient, and homecare. The outpatient condition is a complex environment where the patients flow is unpredictable (Ganguly et al., 2014), patients may fail to show up for their appointment (LaGanga and Lawrence, 2007), patients' appointments might be delayed due to logistical issues (Santibanez et al., 2012), which means that maintaining stable nurse-patient ratios is not feasible (Denton, 2013). A major purpose in outpatient environments is to help the clinic manager assign patients to available nurses in order to provide minimal waiting time for patients (Sevinc et al., 2013, Gocgun and Puterman, 2014, Liang and Turkcan, 2015). NPA in an inpatient environment faces different challenges. Generally, patients in hospitals require more comprehensive care activities, such as a greater number and variety of direct care tasks (e.g., medication administration), as well as more comprehensive and regularly- 
scheduled assessment and monitoring (Spence et al., 2006). Further, patients in inpatient settings will stay in a unit for more than one shift, which increases the complexity of care management and requirements for care coordination (Needleman et al., 2011). Surges in demand are managed differently in inpatient settings, as compared to outpatient contexts. In most hospitals, the N-P Ratio is typically fixed within a certain range by policy to maintain safe staffing standards. In periods of high patient load exceeding the capacity of scheduled nursing staff, a nurse manager cannot simply assign more patients to nurses in a unit; staff assignment policies disallow this, since short-term effects of overwork include increases in care errors, and long-term overwork conditions lead to increased levels of burnout and turnover among nursing staff (Lang et al., 2004).

Several factors have been studied as drivers of nurse-patient assignments in inpatient settings, such as the number of patients needing care (Schaus et al., 2009), the location of patients across the unit (Mullinax and Lawley, 2002), variation of diagnoses and special requirements among patients (Sundaramoorthi et al., 2009), and predicted length of stay (Punnakitikashem et al., 2006). Other considerations include the amount of direct care (nurse time spent directly on patient care, such as medication administration) and indirect care (nurse time spent on other tasks, such like documentation) required by individual patients (Mullinax and Lawley, 2002), the experience and education level of nurses scheduled in a shift (Sundaramoorthi et al., 2009), and patient acuity levels (Mullinax and Lawley, 2002, Schaus et al., 2009, Sir et al., 2015). 
Acuity and Workload Balance in NPA

Acuity is a critical factor to determine nurse-patient assignment (Leiter et al., 1998, Van Slyck and Johnson, 2000, Rogowski et al., 2013, Harper and McCully, 2007). One of the most common factors used in NPA to represent the workload of providing patient's care is acuity (Latimer et al., 2009, Myny et al., 2012). Acuity is a numeric score describing an overall evaluation of the patient's condition based on patient classification systems (Latimer et al., 2009). These systems use several broad considerations, such as diagnoses, the complexity of medications to be administered, the complexity of care procedures required, education level required from staff, and psychosocial issues, to develop a quantitative acuity score (Harper and McCully, 2007). Most commonly in nursing operations, patient acuity is estimated by the patient's time demand on the nursing staff and the complexity of care required (Alghamdi, 2016). In more detailed NPA studies, patient acuity scores were generated by patient classification systems. For instance, acuity for newborns was measured based on fourteen dimensions of patient diagnosis and care complexity (Mullinax and Lawley, 2002, Schaus et al., 2009). In another example, nurses used the QuadMed patient classification system to generate patient acuity scores. This system combines twenty-six different patient acuity indicators, and generates numeric scores (Sir et al., 2015).

A higher acuity score may imply a greater number of nursing orders, perhaps less common or more difficult care procedures, or more frequent visitation requirements and greater vigilance (Brennan and Daly, 2009). Most common nursing care activities (e.g., medication administration, assessments, and documentation) must follow well-defined 
methods for care delivery from standard guidelines (McIntosh et al., 2014). The defined nature of these tasks, which are critical for patient recovery (Auerbach et al., 2013), also make these acuity measures amenable for use in decision-making.

\section{Subjective Workload and the Role of Task Familiarity}

While quantitative factors provide a strong basis for NPA decision-making, many such approaches ignore factors such as uncertainty in patient conditions and variations in individual care needs, because they are more subjective in nature and difficult to quantify (Punnakitikashem et al., 2006, Punnakitikashem et al., 2008). Many complex jobs (Zur Muehlen, 2004, Kittur et al., 2013, Dong et al., 2011), including nursing (Cordery et al., 2010), contain both objective work and subjective work, where subjective work requires addressing unpredictable factors and necessitates the application of a worker's individual judgment and creative thinking (Weston et al., 2006, Griffin et al., 2007).

Subjective workloads require the application of qualitative factors in decisionmaking or judgements, e.g., give employee autonomy to deal with complex tasks (Cordery et al., 2010), allow flexible workflow arrangement (Hornung et al., 2008) and assign familiarity tasks to people in new product development (Reijers et al., 2007). The subjective workload for nurses includes recognizing unexpected changes in patient conditions, which may necessitate calls for emergency procedures, make interventions according to their own judgment, and anticipate upcoming medical orders or care activities (Buerhaus et al., 2005). These activities require nurses to use their critical thinking abilities (Brunt, 2005). These critical thinking abilities in care delivery require a nurse to practice creatively, make judgments with logic, and provide solutions in a complex environment 
(Simpson and Courtney, 2002). Ignoring such factors in a nurse's job can result in negative impacts on nursing performance, including errors in patient care and higher burnout rates (Garrett and McDaniel, 2001).

Work familiarity (e.g., team familiarity and task familiarity) can benefit individual task performance and team interaction in dynamic working fields where subjective workloads are common, such as software development or emergency room care (Reijers et al., 2007, Certa et al., 2009). Previous studies showed that team familiarity could improve team interaction, which leads to better speed and quality of tasks. For example, dynamic software development tasks require cooperation among large numbers of people who are often geographically dispersed, and team familiarity could have a positive effect on performance to overcome the inherent cooperation and communication uncertainties in such scenarios (Harrison et al., 2003, Espinosa et al., 2007). Continuity of care relationships between patients and nurses could benefit the care transmission and the communication between patients and nurses (Austin et al., 1999, McDonald et al., 2007).

Task familiarity can impact individual task performance, since every task needs a different workflow, equipment setting, and environment setting (Goodman and Leyden, 1991), and allow employees to directly retrieve knowledge of different tasks from memory, which reduces task uncertainty in a complex working environment (Lim et al., 1996). The policy of continuity of care, which gives priority to assigning nurses to patients they have cared for previously (Lanzarone and Matta, 2014), can provide task familiarity in uncertain situations where different patients have different requirements and conditions (Allen, 2015) 


\section{Continuity of Care in NPA}

Nurse-to-patient assignment (NPA) under continuity of care (refer to CC) consists of assigning each patient to a nurse they have been assigned in a previous shift during the current hospital stay, chosen among the compatible ones, in order to minimize the number of staff handovers during a patient length of stay. CC task assignment considers the history of previous and current provider-care encounters, and links such relationships to future care (Waibel et al., 2011).

Continuity of care has been considered in healthcare settings that offer multiple episodes of contact with the same patient, such as home care health or primary care (PC) settings (Liu et al., 2018). There are also studies of continuity of care between hospital specialists and PC, the key concern here being care coordination. To the best of our knowledge, no studies have investigated continuity of care in inpatient settings.

It is important to consider the continuity of care in inpatient settings for a number of reasons. First, the $\mathrm{CC}$ assignment holds the possibility of decreasing uncertainty in care delivery, improving the quality of care. Nurses will meet the same patients they have cared for in previous shifts, with greater familiarity between nurses and patients (Allen, 2015). Studies have shown significant benefits associated with CC task assignment: familiarity with the patient is linked to awareness of very subtle changes in the patient status and timely response to patient deterioration (Massey et al., 2017). Secondly, continuity of care relationships between patient and nurse could benefit the care transmission and the communication between patients and nurses (Austin et al., 1999, McDonald et al., 2007) leading to improved patient satisfaction. Patients and their 
families will benefit from additional familiarity, since they could predict which caregiver will serve in the future and the potential care quality to be expected from the caregiver (Haggerty et al., 2003). Primary care patients' experiences showed that CC assignments were associated with better care coordination as perceived by relatives (Bodenheimer, 2008).

In sum, greater continuity in relationships between patients and care providers could help with timely care delivery, timely capture of patients' medical histories and other requirements, and care coordination across providers and settings (Schoen et al., 2009). Therefore, continuity of care a valid factor for the nurse-patient assignment (Plover, 2017). A key concern in including CC in NPA is the impact on operational efficiency (Lanzarone and Matta, 2014). While not yet studied in the literature, discharge related tasks are gaining importance with the goal of reducing hospital readmission rate. Continuity of care, in which more relationship can be developed between nurse, patient, and patient's family, holds the potential to increase the effectiveness of discharge education, although this need to be verified in future work. 


\section{Chapter 3: Problem Description and Research Method \\ Problem Description}

The nurse-patient assignment problem occurs within a broader context in which managers make three types of decisions: first, requirements planning, based on a workload model leading to nurse hiring decisions; second, scheduling decisions, determining on how many nurses to schedule for each shift and how each nurse works over the scheduling horizon; third, a real-time assignment to actual patients, with necessary adjustments to accommodate actual demand (Wright et al., 2006).

This paper considers an inpatient setting in which scheduling decisions have been made a priori, i.e., the number of nurses allocated to the unit and to each shift has been determined. Also predetermined is the scheduling pattern for how each nurse works over the scheduling horizon: either 3 or 4 consecutive days, and either day or night shift. Each nurse will work 14 days per month, which fits common nurse scheduling scenarios (Azaiez and Al Sharif, 2005).

We focus on the NPA problem, the assignment of nurses to specific patients at the beginning of the shift. Building on previous NPA research, we focus on the inherent tradeoff between workload balance and continuity of care, with the objective of helping clinical decision makers implement continuity of care with minimal impact on workload balance. Hence, the performance criteria guiding the NPA decision are workload balance and continuity of care. As a measure of workload, we consider patient acuity, a widelyused numeric measure of the seriousness of a patient's condition (based on diagnosis, medications, and other pertinent information) (Harper and McCully, 2007). The models 
seek to minimize the acuity imbalance across all nurses in a shift in order to ensure that no nurse is overworked, and that each nurse has sufficient time to provide quality care to each patient. To operationalize this, we would like to minimize the cumulative departure from average patient acuity across all nurses in a shift. Simultaneously, we would also like to assign as many patients as possible to nurses based on previous care relationships, i.e., nurses who have cared for them in prior shifts. Ideally, patients would be assigned a single nurse during the length of stay, however, that is not feasible since the length of stay for inpatient admissions typically spans multiple shifts and nurses do not work contiguous shifts. Still, the decision model should seek assignments that minimize the number of nurses that care for the same patient.

The baseline case includes a set of assumptions based on organizational and contextual factors that are typical in inpatient hospital settings. Patients have varying levels of acuity and the level of acuity varies during the patient length of stay. The acuity levels are drawn from a normal distribution with a mean of 0.5 and vary in the range [0.1-0.95. Second, nurses work a 12-hour shift covering either the day or night shift; in reality, nursing shift length may vary across hospitals. Third, we schedule nurses with an assumption of a 1:6 N-P Ratio for expected patient loads. Fourth, we assume that all nurses are interchangeable (i.e., no special skills are required for any patients). Fifth, recognizing that inflexible nursing patient ratios respond poorly when it is necessary to adjust to real demand leading systematically to overstaffing or understaffing (Yankovic and Green, 2011) we allow the decision model to patient loads that exceed the desired N-P Ratio at a penalty. 


\section{Problem Example}

This study was motivated by the question of whether it might be possible to blend quantitative and qualitative goals in multi-criteria assignment policy. Our intuition was that such a scheme would enable us to better understand the tradeoffs associated with these goals. We present a simple example of these potential tradeoffs motivated by the nursepatient assignment problem in an inpatient hospital setting. In this scenario, we would like to pursue a qualitative goal of assigning patients to nurses based on prior care relationships. As a quantitative goal, we consider patient acuity, a widely-used numeric measure of the seriousness of a patient's condition (based on diagnosis, medications, and other pertinent information) (Harper and McCully, 2007). In this scenario, we would like to minimize the acuity imbalance across all nurses in a shift in order to ensure that no nurse is overworked, and that each nurse has sufficient time to provide quality care to each patient. To operationalize this, we would like to minimize the cumulative departure from average patient acuity across all nurses in a shift. Simultaneously, we would also like to assign as many patients as possible to nurses who have cared for them in prior shifts. Obviously, there is a tradeoff between the goal to balance patient acuity across all nurses and to assign patients to nurses based on previous care relationships. Let us consider an example set of patients across a set of shifts, as shown in Table 1, which shows each patient's acuity for each shift. 


\begin{tabular}{|l|l|l|l|}
\hline Patient/Acuity & Shift 1 & Shift 2 & Shift 3 \\
\hline Smith & 0.75 (new admit) & 0.8 & 0.8 \\
\hline Jones & & & 0.9 (new admit) \\
\hline Singh & 0.8 & 0.9 & 0.7 \\
\hline Chen & & 0.8 (new admit) & 0.6 \\
\hline Hardy & 0.6 & 0.5 & 0.4 \\
\hline Acosta & & & 0.35 (new admit) \\
\hline Miller & 0.5 & 0.3 (discharge) & \\
\hline Carter & 0.3 (discharge) & & \\
\hline
\end{tabular}

\section{Table 1: Patients' status and acuity across shifts}

Table 2 shows the shift work schedule for nurses. Shifts 1 and 2 represent prior shifts, while Shift 3 represents the current shift, for which we need to assign nurses to patients. $\mathrm{A}^{\text {'*' }}$ indicates that a nurse is working in a shift.

\begin{tabular}{|l|l|l|l|}
\hline Nurse Schedule & Shift 1 & Shift 2 & Shift 3 \\
\hline Sam & $*$ & & $*$ \\
\hline Jean & $*$ & & $*$ \\
\hline Carol & & $*$ & \\
\hline Anna & & $*$ & \\
\hline
\end{tabular}

Table 2: Nurse schedule across three shifts

Table 3 shows the nurse-patient assignments across prior shifts. A ' $*$ ' indicates that a nurse has cared for a patient in a previous shift.

\begin{tabular}{|l|l|l|l|l|l|l|l|l|}
\hline $\begin{array}{l}\text { Prior-care } \\
\text { Relationships }\end{array}$ & Smith & Jones & Singh & Chen & Hardy & Acosta & Miller & Carter \\
\hline Sam & $*$ & & & & & & $*$ & \\
\hline Jean & & & $*$ & & $*$ & & & $*$ \\
\hline Carol & & & $*$ & $*$ & & & & \\
\hline Anna & $*$ & & & & $*$ & & $*$ & \\
\hline
\end{tabular}

Table 3: Nurse-patient relationships before Shift 3

First, we note that newly admitted patients have no prior-care relationships. In this case, we have two new patients, Jones and Acosta, for Shift 3. For these patients, the relationship-based assignments are not possible. Second, some patients may have prior care 
relationships, but the corresponding nurses may or may not be scheduled to work in the current shift. In this case, we have one prior patient, Chen, without a prior care relationship with nurses working in Shift 3. Here as well, the relationship-based assignments are not feasible. Third, nurses with prior care relationships may be assigned to the current shift, but the patients they cared for may have been discharged. In this case, we have one prior patient, Miller, who was discharged before Shift 3 (but had a care relationship with currentshift nurse Sam). Here, too, the relationship-based assignment cannot be applied.

Let us now consider what happens in the case where we pursue an approach (Assignment I) based purely on minimizing acuity imbalances. Here, we assign Smith, Singh, and Acosta to Jean, and Jones, Chen, and Hardy to Sam. In this case, we have minimized acuity imbalance across the nurses, but only Singh has a prior care relationship with Jean (33\% of prior care relationships for Jean, and 0\% of care relationships for Sam).

Next, we consider what happens when we first assign patients to nurses based on prior care relationships (Assignment II). Here, we assign Smith to Sam (33\% of prior care relationships for Sam), and Singh and Hardy to Jean (66\% of prior care relationships for Jean) based on the prior care relationships before Shift 3. We note that these initial assignments result in acuity assignments as well, giving nurses with prior-care relationships initial acuity workload scores (where the workload score for the purposes of this example is a simple sum of assigned patients' acuity): Sam has a workload score of 0.8 , while Jean's workload score is 1.1 , and some patients remain unassigned. If we assign the remaining patients to nurses based on acuity, considering the acuity scores assigned based on prior relationships, then we will make the following assignments: Jones and 
Acosta are assigned to Sam, while Chen is assigned to Jean. This results in acuity scores as follows for each nurse: 1.70 for Jean and 2.05 workload for Sam. Here, there is a marginal increase in acuity imbalance over the acuity-only case, 0.05 vs. 0.35 ; however, we were also able to assign $50 \%$ of patients ( 3 of 6 ) to nurses based on prior care relationships at relationship-based case compared to $17 \%$ of patients ( 1 of 6 ) to nurses based on prior care relationships at acuity-only case. In this scenario, for a penalty in workload imbalance, we were able to gain a substantial percentage of relationship-based assignments, with their attendant benefits. In other word, this is a tradeoff between workload balance and continuity of care. The detailed information of these two assignments is shown in Figure 1 below.

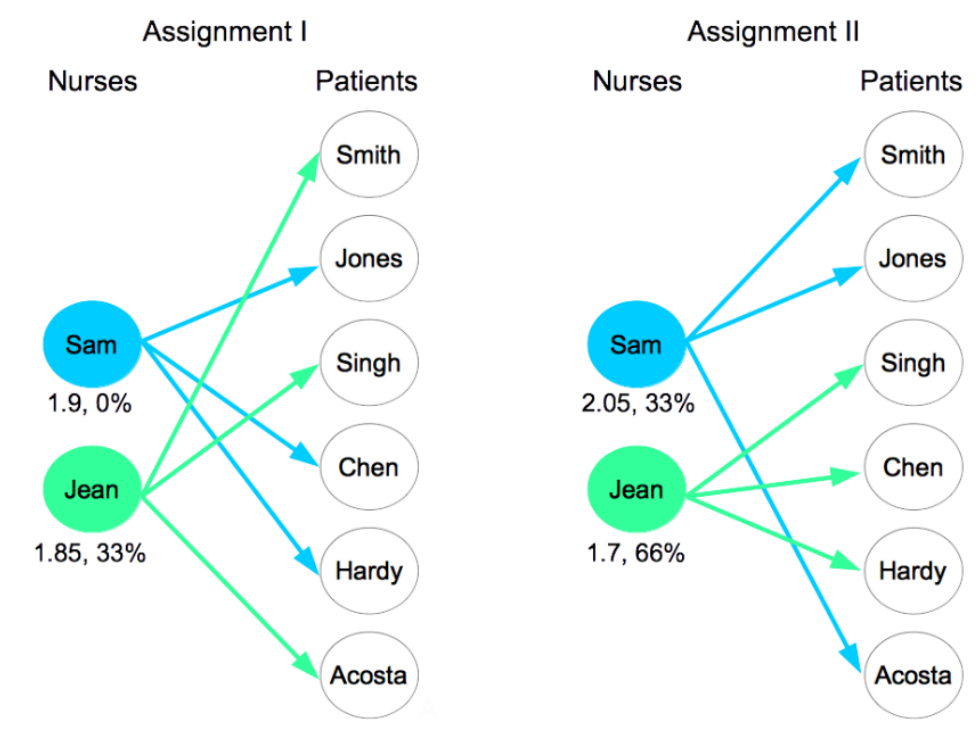

Figure 1: Two different NPA policies for the same patient census

There are two numbers associated with each nurse; the first representing the total workload score and the second representing the percentage (continuity of care) from all assigned patients. 


\section{Research Model}

\section{Notations}

In this section, we present a model of workload in hospital shift assignments and a summary of the notation we will use through the remainder of the paper. Further, we present a set of algorithms that demonstrate how we can blend both quantitative and qualitative goals in a workload balancing method. We present these algorithms in the context of acuity and relationship-based assignment.

\begin{tabular}{|c|c|}
\hline Notation & Meaning \\
\hline$C$ & $\begin{array}{l}\text { A sequence of shifts contains last shift } c \text {-1, and current shift } c . C= \\
\{\mathbf{1} \ldots \boldsymbol{c}\} \text {. }\end{array}$ \\
\hline $\boldsymbol{c}$ & Current shift number \\
\hline $\boldsymbol{h}_{j}$ & Nurse working in the unit with ID $\boldsymbol{j}$ \\
\hline$\vec{H}$ & $\begin{array}{l}\text { Set of nurses }\left(\boldsymbol{h}_{\boldsymbol{j}}\right) \text { with ID } \boldsymbol{j} \text { in the pool of nurses working in the unit, } \\
\text { such that } \boldsymbol{h}_{\boldsymbol{j}} \boldsymbol{\epsilon} \boldsymbol{H}\end{array}$ \\
\hline $\boldsymbol{H}_{\boldsymbol{c}}$ & $\begin{array}{l}\text { Set of nurses }\left(\boldsymbol{h}_{\boldsymbol{j}}\right) \text { to represent nurses working in current shift } \boldsymbol{c} \text {, such } \\
\text { that } \boldsymbol{H}_{\boldsymbol{c}} \subseteq \boldsymbol{H}\end{array}$ \\
\hline$p_{i}$ & Each patient $\left(\boldsymbol{p}_{\boldsymbol{i}}\right)$ ID $\boldsymbol{i}$ in a shift. \\
\hline $\boldsymbol{e}_{\boldsymbol{i}}$ & Shift number of admissions for each patient $\left(\boldsymbol{p}_{\boldsymbol{i}}\right)$ \\
\hline$f_{i}$ & Shift number of discharges for each patient $\left(\boldsymbol{p}_{\boldsymbol{i}}\right)$ \\
\hline$t_{p i}$ & Patient $\boldsymbol{i}$ normalized acuity in a shift, $\boldsymbol{t}_{\boldsymbol{p} \boldsymbol{i}}$ is in the range $\left[\begin{array}{lll}\mathbf{0} & \ldots & \mathbf{1}\end{array}\right]$. \\
\hline $\boldsymbol{P}$ & $\begin{array}{l}\text { Set of tuples }\left(\boldsymbol{p}_{\boldsymbol{i}}, \boldsymbol{e}_{\boldsymbol{i}}, \boldsymbol{f}_{\boldsymbol{i}}\right) \text { for all patients' records in all shifts, such that } \\
\boldsymbol{P}=\left\{\left(\boldsymbol{p}_{\mathbf{1}}, \boldsymbol{e}_{\mathbf{1}}, \boldsymbol{f}_{\mathbf{1}}\right) \ldots\left(\boldsymbol{p}_{\boldsymbol{i}}, \boldsymbol{e}_{|\boldsymbol{P}|}, \boldsymbol{f}_{|\boldsymbol{P}|}\right)\right\} \text {. }\end{array}$ \\
\hline$\overline{P_{c}}$ & $\begin{array}{l}\text { Set of tuples }\left(\boldsymbol{p}_{\boldsymbol{i}}, \boldsymbol{t}_{\boldsymbol{p} \boldsymbol{i}}\right) \text { for patients' records in current shift, such that } \\
\boldsymbol{P}_{\boldsymbol{c}}=\left\{\left(\boldsymbol{p}_{\mathbf{1}}, \boldsymbol{t}_{\boldsymbol{p} \mathbf{1}}\right) \ldots\left(\boldsymbol{p}_{\left|\boldsymbol{P}_{\boldsymbol{c}}\right|} \boldsymbol{t}_{\left|\boldsymbol{P}_{\boldsymbol{c}}\right|}\right)\right\} \text {. }\end{array}$ \\
\hline$W_{c}$ & $\begin{array}{l}\text { Set of working packages }\left(\boldsymbol{w}_{\boldsymbol{k}}\right) \text { to store patients' information for nurses } \\
\boldsymbol{h}_{\boldsymbol{j}} \text { in shift } \boldsymbol{c} \text {, such that } \boldsymbol{W}_{\boldsymbol{c}}=\left\{\boldsymbol{w}_{\mathbf{1}} \ldots \boldsymbol{w}_{\boldsymbol{k}}\right\} \text {. }\end{array}$ \\
\hline$w_{k}$ & $\begin{array}{l}\text { Working package }\left(\boldsymbol{w}_{\boldsymbol{k}}\right) \text { to store patients' information in a shift, } \boldsymbol{k}= \\
\left\{\mathbf{1} \ldots\left|\boldsymbol{H}_{\boldsymbol{c}}\right|\right\}\end{array}$ \\
\hline $\operatorname{acu}\left(w_{k}\right)$ & Workload for working package $\boldsymbol{w}_{k}$, where $\boldsymbol{a c u}\left(\boldsymbol{w}_{\boldsymbol{k}}\right)=\sum_{\boldsymbol{i}=\mathbf{1}}^{|\boldsymbol{u}|}\left(\boldsymbol{t}_{p i}\right)$. \\
\hline $\boldsymbol{u}$ & Average number of patients per nurse in a shift, where $\boldsymbol{u}=\left\lceil\left|\boldsymbol{P}_{\boldsymbol{c}}\right| /\left|\boldsymbol{H}_{\boldsymbol{c}}\right|\right\rceil$ \\
\hline$Z_{j}$ & The total workload of nurse $\boldsymbol{h}_{\boldsymbol{i}}$ in a shift \\
\hline
\end{tabular}




\begin{tabular}{|c|c|}
\hline$a_{c}$ & $\begin{array}{l}\text { The average workload of each nurse in a shift, where } \boldsymbol{a}_{\boldsymbol{c}}= \\
\sum_{\boldsymbol{i}=\mathbf{1}}^{\left|\boldsymbol{P}_{\boldsymbol{c}}\right|}\left(\boldsymbol{t}_{\boldsymbol{p} \boldsymbol{i}}\right) /\left|\boldsymbol{H}_{\boldsymbol{c}}\right|\end{array}$ \\
\hline$s_{i j}$ & $\begin{array}{l}\text { Represent the historic care times where } \boldsymbol{h}_{j} \text { cared for } \boldsymbol{p}_{\boldsymbol{i}} \text { before, } \boldsymbol{s}_{\boldsymbol{i j}}= \\
\sum_{\boldsymbol{c - \boldsymbol { e } _ { i }}}^{\boldsymbol{c - 1}}\{\mathbf{1}\} \text { where } \boldsymbol{h}_{\boldsymbol{j}} \rightarrow \boldsymbol{p}_{\boldsymbol{i}} \text { in previous }\left(\boldsymbol{c}-\boldsymbol{e}_{\boldsymbol{i}}\right) \text { shifts }\end{array}$ \\
\hline$S_{c}$ & $\begin{array}{l}\text { Set of tuples }\left(\boldsymbol{p}_{\boldsymbol{i}}, \boldsymbol{S}_{\boldsymbol{i}}, \boldsymbol{h}_{\boldsymbol{j}}\right) \text { to represent prior care relationships among } \\
\text { nurses and patients in current shift, such that } \boldsymbol{P}= \\
\left\{\left(\boldsymbol{p}_{\mathbf{1}}, \boldsymbol{s}_{\mathbf{1 1}}, \boldsymbol{h}_{\mathbf{1}}\right) \ldots\left(\boldsymbol{p}_{\left|\boldsymbol{P}_{\boldsymbol{c}}\right|}, \boldsymbol{S}_{\left|\boldsymbol{P}_{c}\right| *\left|\boldsymbol{H}_{c}\right|}, \boldsymbol{h}_{\left|\boldsymbol{H}_{c}\right| \mid}\right)\right\} \text {. }\end{array}$ \\
\hline$Q_{c}$ & $\begin{array}{l}\text { Array } \boldsymbol{Q}_{c}=\left\{\mathbf{1} \ldots\left|\boldsymbol{P}_{c}\right|\right\} \text {, where each element is defined as } 1 \text { if the } \\
\text { corresponding patient was assigned based on a prior-care relationship, } \\
\text { otherwise } 0 .\end{array}$ \\
\hline$v_{j m}$ & Historic workload for nurse $\boldsymbol{h}_{\boldsymbol{j}}$ in previous $\boldsymbol{m}$ shifts \\
\hline$V_{c}$ & $\begin{array}{l}\text { Set of tuples }\left(\boldsymbol{h}_{\boldsymbol{j}}, \boldsymbol{v}_{\boldsymbol{j} \boldsymbol{m}}\right) \text { to represent the historic average workload for } \\
\text { each nurse in current shift }\end{array}$ \\
\hline$d$ & $\begin{array}{l}\text { Threshold of acuity } \boldsymbol{t}_{\boldsymbol{p} i} \text { to be considered in care relationship assignment, } \\
\boldsymbol{d} \text { is in the range }\left[\begin{array}{lll}\mathbf{0} & \ldots & \mathbf{1}\end{array}\right] .\end{array}$ \\
\hline
\end{tabular}

\section{Table 4: Summary of Notation}

We describe a model for the information needed in the nurse-patient workload assignment problem. In Table 4, we present a summary of the notation used throughout the remainder of the paper. We use $C$ to represent a sequence of shifts, where $c$ represents the shift number of the current working shift. Each nurse is represented by an identifier $h_{j}$, where $H$ is pool of nurses working in a unit, and $h_{j} \in H . H_{c}$ is a set of nurses $\left(h_{j}\right)$ nurses working in current shift $c$, where $H_{c} \subseteq H$. We use $p_{i}$ to represent a patient by id $i, e_{i}$ to represent a patient's shift of admission, $f_{i}$ to represent a patient's shift of discharge, and $t_{p i}$ to represent patient's normalized acuity, which is in the range $[0 \ldots 1]$. We use $P$ as a set of tuples $\left(p_{i}, e_{i}, f_{i}\right)$ to represent all patients' records in all shifts, and $P_{c}$ as a subset of tuples $\left(p_{i}, t_{p i}\right)$ that represents patients' acuity in current shift $c$.

$W_{c}$ is a set of working packages in a shift, where $w_{k} \in W_{c}$. We used $w_{k}$ to represent the working package of each nurse in a shift. We count the summary of the patients' acuity 
(denoted by $\operatorname{acu}\left(w_{k}\right)$ ) for working package $w_{k}$ in each assignment round until all $p_{i}$ are assigned to nurses $h_{j}, a c u\left(w_{k}\right)=\sum_{i=1}^{|u|}\left(t_{p i}\right)$, where $p_{i} \in w_{k}$. Then we employ $u$ as the average number of patients per nurse in current shift, where $u=\left\lceil\left|P_{c}\right| /\left|H_{c}\right|\right\rceil$. The total workload of nurse $j$ in a shift is represented by $z_{j}$. The average workload of each nurse in a shift is represented by $a_{c}$, where $a_{c}=\sum_{i=1}^{\left|P_{c}\right|}\left(t_{p i}\right) /\left|H_{c}\right|$.

For relationship-based assignments, we note that it is possible that there may be multiple nurses with prior-care relationships, so we need a means of selecting one priorcare relationship over others. Here, we assign the patient to the nurse with the largest count of prior-care shifts (or, given equal counts, the most recent prior-care shift). To determine this, we access the set of prior-care relationships $S_{c}$ to calculate $s_{i j}$. Specifically, $s_{i j}=$ $\sum_{c-e_{i}}^{c-1}\{1\}$, where $h_{j} \rightarrow p_{i}$. This captures a count of shifts in which a nurse $h_{j}$ has cared for a patient $p_{i}$ since $p_{i}$ arrived in the unit (i.e., over the previous $\left(c-e_{i}\right)$ shifts), which is stored in array $Q_{c}$.

For workload balanced assignments, $v_{j m}$ represents the average historic workload for nurse $h_{j}$ in the previous $m$ shifts; $v_{j m}=\sum_{m=c-y}^{c}\left(z_{j}\right) / m$. We use $V_{c}$ to store a set of tuples containing historic average workload $V_{j m}$ for each nurse $h_{j}$ in shift $c$, then assign $w_{k}$ to $h_{j}$ based on the differences in $v_{j m}$.

\section{Policies Overview}

To demonstrate the possibility of blending quantitative and qualitative goals, we present a method for the nurse-patient assignment based on both acuity and relationship continuity goals. We aim at a heuristic approach for a near-optimal solution that can be determined 
with minimal computation. It is possible to solve this problem as an integer programming problem; however, solvers are not guaranteed to produce the solutions in a short time frame, which is needed in a hospital environment.

We call our method the continuity of care (CC) - workload balanced (WB) policy. In this hybrid algorithm, the available patients in a shift will be classified into a priorpatient group (in hospital for more than one shift) and a new-patient group (just admitted into the unit). For the prior-patient group, we track their previous care records, and attempt to assign them to available nurses who have cared for them before. Based on the length of the patients' stays and the nurses' schedules, we cannot necessarily assign all prior-care patients based on prior-care relationships. For the new-patient group and any prior patients not assigned based on prior-care relationships, we assign these patients to nurses to balance acuity as far as possible in the shift.

To demonstrate the effectiveness of this hybrid policy we contrast its performance with three other assignment methods. First, we developed a WB policy. The WB policy attempts to balance the workload among available nurses in several shifts. Second, we developed a patient number balanced policy (which we refer to as the NB case), in which patients are assigned to nurses without a quantitative or a qualitative goal; we simply ensure that we balance the number of patients per nurse to the extent possible. Finally, we developed a CC-NB policy. The CC-NB policy works much like the CC-WB policy, except that patients who cannot be assigned based on prior care relationships are assigned randomly, rather than based on acuity. 
In the remainder of the section, we describe the $\mathrm{WB}$ policy and the $\mathrm{CC}-\mathrm{WB}$ policy. We omit a detailed description of the NB policy, since it is clear without further detail. We also omit a detailed description of the CC-NB policy, because it is similar to the CC-WB policy (using random assignment rather than acuity for patients without prior-care relationships).

\section{The WB Policy}

In the WB policy, we try to minimize acuity workload imbalance across the set of nurses working in a shift, i.e., we purse purely objective goals in workload distribution.

In this approach, we first create several empty working packages $w_{k}$ for the number of nurses $\left|H_{c}\right|$ in a shift. We need $\left[\left|P_{c}\right| /\left|H_{c}\right|\right]$ number of rounds to assign patients $p_{i}$ to working packing packages $w_{k}$. In each round, we order all working packages $w_{k}$ in ascending order by their total workload $a c u\left(w_{k}\right)$. We then order all unassigned patients $p_{i}$ in descending order by their acuity $t_{p i}$. We then assign the patients $p_{i}$ to working packages $w_{k}$ according to these orderings. After all patients are assigned to working packages, the nurse with the lowest historic workload $v_{j m}$ from the previous $m$ shifts is assigned the working package with the highest workload, while a nurse with highest historic workload $v_{j m}$ from previous $m$ shifts is given the working package with the lowest workload.

\begin{tabular}{cl}
\hline Algorithm 1: WB policy \\
\hline 1. & Input current shift number $c$ \\
2. & Input a set of $H_{c}$ \\
3. & Input a set of $P_{c}$ \\
4. & Input a set of $V_{c}$ \\
5. & for $k=1$ to $\left|H_{c}\right|$ \\
6. & create $w_{k}, w_{k}$ is empty \\
\hline
\end{tabular}


7. end for

8. sort $P_{c}$ by descending order the $p_{i}$ with $t_{p i}$

9. for number of round $=1$ to $u / /$ total rounds

10. for $k=1$ to $\left|H_{c}\right|$

11. if $\left|P_{c}\right|>0$

12. $p_{\max }=p_{i}$ with $\max \left(t_{p i}\right)$

13. assign $p_{\max }$ to $w_{k}$

14. remove $p_{\max }$ from $P_{c}$

15. end if

16. end for

17. sort $w_{k}$ by ascending order of $\operatorname{acu}\left(w_{k}\right)$

18. end for

19. sort $h_{j}$ by ascending order of $v_{j m}$ from $V_{c}$

20. for $k=1$ to $\left|H_{c}\right|$

21. set $h_{j} \rightarrow w_{k}$ mapping //finish the n-p assignment

22. end for

23. return all $w_{1}$ to $w_{\left|H_{c}\right|}$ where $h_{j} \rightarrow w_{k}$ mapping //output of $\mathrm{n}-\mathrm{p}$ assignment

Algorithm 1 describes this method in more detail. Inputs are current shift number $c$; nurse information with personal ID $h_{j}$ from $H_{c}$, patient information with personal ID $p_{i}$ from $P_{c}$ and current acuity $t_{p i}$; and average historic workload $v_{j m}$ from $V_{c}$. We need all these inputs to be part of our workload balanced nurse-patient assignment (Alg. 1, lines $1-4)$.

We then initialized working package structure $w_{k}$ to fit the number of nurses $\left|H_{c}\right|$ in the current shift (Alg. 1, lines 5-7). We then sort all patients by descending order their current acuity (Alg. 1, line 8). For each $(u, k)$ pair, if the number of patients $\left|P_{c}\right|>0$, we assign the patient with highest current acuity $t_{p i}$ to the working package $\mathrm{w}_{\mathrm{k}}$ with lowest total acuity $\operatorname{acu}\left(w_{k}\right)$, and continue this order-assign process until all current patients have been assigned to working packages. After these assignments, we sort all the working packages by ascending order based on total acuity $a c u\left(w_{k}\right)$ (Alg. 1, lines 9-18). 
We sort nurses by descending order of their average historic working acuity $v_{j m}$ (Alg. 1 , line 19). For nurses in the current shift, we assign a nurse to each working package based on these orderings (Alg. 1, lines 20-22), and output the nurses-patients' assignment with total acuity (Alg. 1, line 23).

\section{The Hybrid CC-WB Policy}

The CC-WB policy seeks to achieve workload balance among available nurses in several shifts, as well as accrue the qualitative benefits available from the continuity of care relationships between nurses and patients. In the proposed policy, we use seven steps to do the NPA assignment.

1. Separate the patients in the current shift into two groups, a new-patient group (with no prior care relationships) and a prior-patient group (with prior care relationships).

2. Consider the nurse-patient care relationships from previous shifts for the priorpatient group.

3. Store care relationships in the set of tuples, which record the patient-nurse care relationship (i.e., count 1 as existed care relationship, while count 0 as no care relationship) from previous shifts.

4. Sort these tuples by descending order of care relationship between nurses and patients

5. Assign the patient with maximum care relationship record with the nurse and meeting the acuity threshold such patient (i.e., patient acuity is bigger or equal to acuity threshold will be considered to use this CC approach). 
6. Move the remaining patients (i.e., patients in the prior-care group who cannot be assigned based on prior-care relationships) to the new-patient group.

7. Used workload balancing function to assign them to each nurse based on the WB approach for the new-patient group.

We separate the patients in the current shift into two groups, a new-patient group (with no prior care relationships) and a prior-patient group (with prior care relationships). For the prior-patient group, we consider nurses-patients care relationships from previous shifts. These care relationships are stored in the set of tuples $S_{c}$, which record the patientnurse care relationship $\left(p_{i}, s_{i j}, h_{j}\right)$ from previous shifts. We sort $S_{c}$ by descending order of $s_{i j}$, and then assign the $p_{i}$ with maximum $s_{i j}$ and meeting the acuity threshold $t_{p i} \geq d$ to the list $B$, which stores the patients' information with maximum care times. We then assign $p_{i}$ in list $B$ to $h_{j}$. For the remaining patients in the prior-care group who cannot be assigned based on prior-care relationships, we move these patients to the new-patient group. For the new-patient group, we used workload balanced function to assign them to each nurse based on the workload $a c u\left(w_{k}\right)$. The algorithm is presented below.

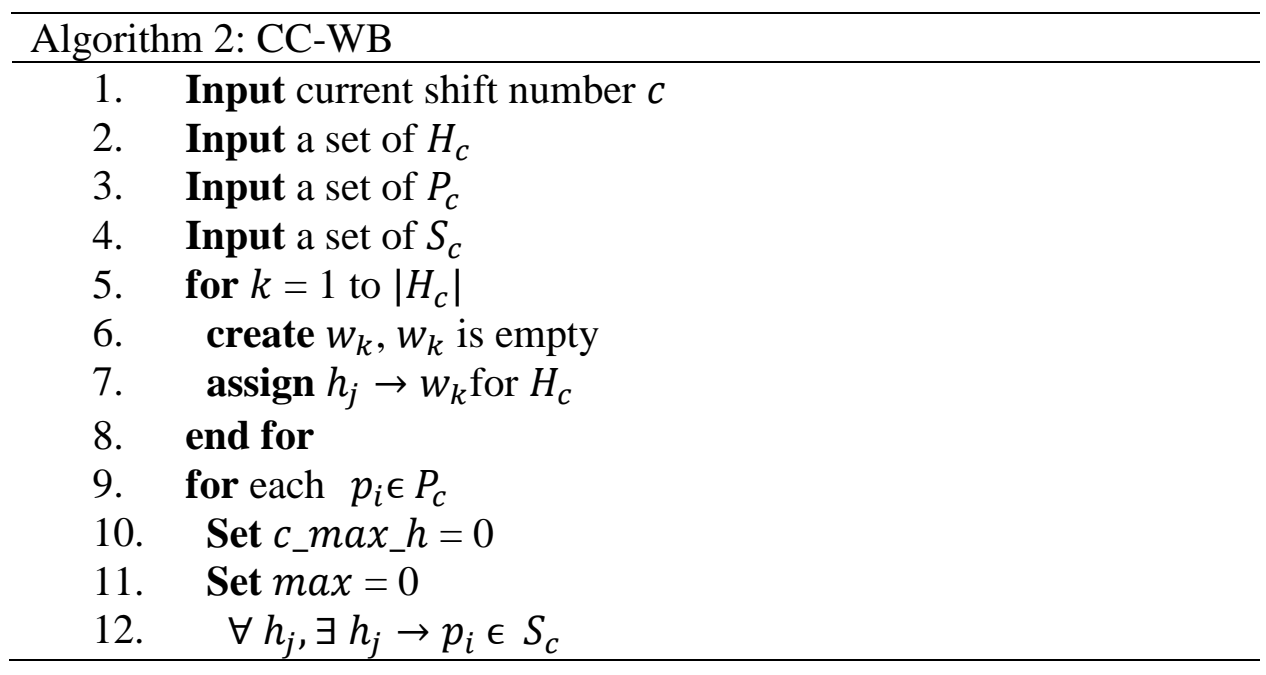


13. if $s_{i j}$ for $h_{j} \rightarrow p_{i}>\max$

14. $\max =s_{i j}$

15. $\quad$ c_max_h $h=j$

16. end if

17. end for

18. If $c \_m a x \_h>0$

19. $\quad$ assign $p_{i} \rightarrow w_{k}$ where $w_{k} \rightarrow h_{j}$

20. remove the assigned $p_{i}$ from $P_{c}$

21. end if

22. end for

23. if $\left|P_{c}\right|>0$

24. sort $P_{c}$ by descending order the $p_{i}$ with $t_{p i}$

25. for $h_{j}, j=1$ to $\left|H_{c}\right|$

26. sort $\operatorname{acu}\left(w_{k}\right)$, by ascending order of $\operatorname{acu}\left(w_{k}\right)$, then by $\left|w_{k}\right|$ for all $h_{j}$ with equal $\operatorname{acu}\left(w_{k}\right)$

27. end for

28. assign $p_{i}$ with $\max \left(t_{p i}\right) h_{j}$ with $\min \left[\operatorname{acu}\left(w_{k}\right)\right]$

29. remove the assigned $p_{i}$ from $P_{c}$

30. end if

Inputs are current shift number $c$; set of nurses working in current shift $H_{c}$ with personal ID $h_{j}$; set of patients $P_{c}$ in current shift with personal ID $p_{i}$ and current acuity $t_{p i}$; and set of historic nurse-patient care relationship $S_{c}$, which holds the carerelationship counts $\left(s_{i j}\right)$ for patient $p_{i}$ with nurse $h_{j}$ in the previous $\left(c-e_{i}\right)$ shifts. We need all these inputs to support the relationship-based nurse-patient assignment (Alg. 2, lines 1-4).

For each nurse in the current shift, we use the perimeter $w_{k}$ to store the patient set assigned to each nurse $h_{j}$. Each working package $w_{k}$ is empty to start (Alg. 2 , lines 5-8). For each patient in the current shift, we create nurse-patient assignments in three stages: (1) separate patients in prior-care and new-patient groups; (2) for prior patient group, we assign patients to nurses based on prior-care relationships; and (3) for new admission 
patient or prior patient who do not have previous care relationship with current nurses, we use the WB function to assign them to nurses (Alg. 2, lines 9-30). We set the parameter $c_{-} m a x_{-} h$ to store current nurses who had previous care relationships with patients (Alg. 2, line 10). We set the parameter $\max$ to store the maximum care times $s_{i j}$ (Alg. 2, line 11). For current nurses who have previous care relationships with patients in we find these nurses where $h_{j} \rightarrow p_{i}$ map with the maximum value of $\left(s_{i j}\right)$ and acuity $t_{p i}$ of $p_{i}$ is greater or equal to $d$. We store these nurses' information into perimeter $c_{-} m a x_{-} h$. If more than one maximum $\left(s_{i j}\right)$ exists, we assign patients to nurses based on the most recent care relationship. (Alg. 2, lines 11-17). If the $c_{-} m_{a x} \_h$ is bigger than zero, we assign the $p_{i}$ to $w_{k}$ for $h_{j}$ based on the historic care relationship. We removed the assigned patients $p_{i}$ from $P_{c}$ (Alg. 2, lines 18-22).

For new admission patients or prior patients who do not have previous care relationships with current nurses in $H_{c}$, we use WB function to assign them to nurses (Alg. 2, lines 23-30). We first sort the remaining patients by descending order their current acuity $t_{p_{i}}$ (Alg. 2, lines 24). We then sort the nurses in the current shift by ascending order their current workload $w_{k}$ (Alg. 2, lines 25-27). We assign the remaining patient with the highest current acuity $t_{p_{i}}$ to the $h_{j}$ with the lowest total acuity $w_{k}$ at each time, and continue this order-assign process until all remaining patients have been assigned (Alg. 2, lines 28-30). 


\section{NB Policy}

The number balancing (NB) policy is subject only to numeric balance, i.e. each nurse has the same number of patients, which are selected randomly. This is a common practice followed by charge nurses in real inpatient hospital settings.

\section{Policy}

Continuity-care (CC) method prioritizes continuity. In this algorithm, the available patients in a shift will be classified into a prior-patient group (in hospital for more than one shift) and a new-patient group (just admitted into the unit). For the priorpatient group, we track their previous care records, and assign them to available nurses who have cared for them before, giving priority to the nurse with the higher care frequency, i.e., the number of shifts they previously cared for this patient. Based on the length of the patients' stays and the nurses' schedules, we cannot necessarily assign all prior-care patients based on prior-care relationships. For the new-patient group and any prior patients not assigned based on prior-care relationships, we assign these patients to nurses based on NB policy ensure numeric balance. 


\section{Chapter 4: Data Simulation}

\section{Simulation Overview}

Simulation models have been used to analyze the performance of heuristics related to job allocation problems (Amoako-Gyampah and Meredith, 1996), and are well suited to represent the complexities of a medical surgery unit (Ferrand et al., 2014). We adopt a simulation methodology adapting to the NPA context. In this section, we describe the simulated operational setting, the performance metrics, and NPA heuristic-based policies. The goal of the simulation is to assess the performance of heuristics that address continuity of care and workload balance objectives in the NPA. The simulation model allows us to explore constraints and options that are challenging to model in the MIP. We can also capture the stochastic characteristics of patients' lengths of stay, which would turn the optimization model further complex.

We start with a simple set of heuristics to understand the nature of the tradeoff between continuity of care and nurse workload balance. These simple heuristics seek to maximize continuity of care. We then explore the performance impact of a heuristic that seeks to break the performance tradeoff in a way that still prioritizes continuity of care. The simulation model enables an understanding of the potential benefits of adopting these scheduling policies. We want to understand the tradeoff space for different assignment solutions, and plan to further explore the impact of contextual factors (e.g., different patient flow, different shift arrangements) to our hybrid approach. 


\section{Baseline Case Setting}

Our model simulates the operation of an inpatient hospital unit setting for four weeks (56 shifts). We consider a unit staffed by 21 nurses, a dimension consistent with previous studies (Goncalves et al., 2007), and nurses work in 12-hour shifts (Leiter et al., 1998, Bushnell et al., 2010). Each nurse is consistently scheduled for either days or nights (i.e., nurses assigned to day shifts consistently work days, and nurses assigned to night shifts consistently work nights) (Scott et al., 2006). Nurses typically work four days a week (Caruso, 2014). The nurse schedule is either 3 consecutive days or 4 consecutive days per week, and each nurse will work 14 days per month.

There are 42 beds in the unit, i.e., the unit can provide care for a maximum of 42 patients in a shift. Based on the literature, we set the nurse-patient ratio at 1:6 for the baseline case (Duffield et al., 2011). The number of patients generated in 30 days is based on the average length of stay, 10 shifts, and the nurse capacity in one month, i.e., the total care shifts (sum for all nurse in a unit) is 294 (21 nurses * 14 shifts) and target nurse-patient ratio of 1:6. Hence, the number of patients admitted to the unit per shift follows a normal distribution around a mean of 2.77 and a standard deviation of 1.70. Each patient stays between 4 and 22 shifts, distributed normally around a mean of 9.07 (Stimpfel et al., 2012, Brodovicz et al., 2013, Pakzad et al., 2014), also consistent with previous studies that show patient length of stay varies between 6 shifts to 14 shifts with an average 10 shifts (Cleary et al., 1991).

Patients are assigned a series of acuity scores during the patient's length of stay in the hospital, and such acuity score changes shift by shift to represent a patient's condition 
changes in the real world. The acuity scores are drawn from a normal distribution with a mean of 0.50 (acuity scores fall in the range [0..1.0]). The normal distribution represents a typical patient condition in a general medical unit, since patients with low acuity (2\%) (less than 0.1 ) tend to be discharged, and patients with high acuity (2\%) (greater than 0.90) tend to be transferred to higher-care units or another hospital.

We created 50 separate simulated datasets, with a combined total of 9,000 simulated patients' records (180 patients per dataset), and 81670 patient-shift entitles, where each patient-shift entity represents an individual instance of the nurse-patient assignment problem. On average, there are 21 patients per shift across all shifts in our data sets. This represents an average nurse-patient ratio of 1:5.56 per shift, which is representative of real-world nurse-patient ratios (Lang et al., 2004).

\section{Performance Metrics}

To measure the amount of acuity imbalance that results from the given workload distribution method, we use the metric defined in the optimization model, the Accumulated Acuity Imbalance (AAI). AAI is calculated as the sum across all nurses and across all shifts, of the absolute difference between each nurse workload in a shift and the average workload per nurse in the same shift. Specifically, we calculate AAI as follows: $A A I=$ $\sum_{1}^{|C|} \sum_{1}^{|H c|}\left|z_{j}-a_{c}\right|$

For the continuity goal, the discrete event simulation model allows us to consider a more granular view of the history of care relationships, and track the percentage of patients assigned to nurses based on prior care relationships. To measure this, we define a metric called Percentage of Continuity of Care (PCC), where PCC is calculated as follows: PCC = 
$\frac{\sum_{c=3}^{|C|} \sum_{g=1}^{|P c|}\left(Q_{g}\right)}{\sum_{c=3}^{|C|}\left|P_{c}\right|}$. We begin to count PCC at shift 3 since no patients will have prior care relationships at the first two shifts.

We use the output from 50 simulation runs to compete for the average AAI and average PCC for each policy. 
Chapter 5: Results

\section{Initial Results}

In this section, we present the results of the baseline model for the simple hybrid heuristic. In our experimental evaluation, we first measure AAI and PCC for each of four simple heuristic methods: CC, WB, CC-WB, and NB. The CC-WB approach is our proposed method for blending continuity of care and workload balance goals. WB attempts to minimize acuity, $\mathrm{CC}$ case represents a scenario where we pursue maximum relationshipbased assignment.

We present the AAI and PCC results in Table 7. Recall, the AAI metric represents the sum of all acuity imbalances (difference from average for each nurse in a shift) across all shifts; we use this metric to demonstrate the accumulated effects in terms of workload imbalance over time. To support continuity of care scheduling (which requires knowledge of prior care relationships), we generated an initial set of two setup shifts representing shifts occurring before the start of our four-week simulation scenarios. In these setup shifts, we used an NB method to assign nurses to shifts, solely to provide the possibility of prior-care relationships across all shifts. We do not report on the metric for continuity of care for these setup shifts.

The four policies (CC, WB, CC-WB, and NB) are designed to achieve different purposes; therefore, the workload distributions of these four policies should be different. We first performed a Levene test to do the manipulation check that should show all four approaches generate different workload distributions (per nurse per shift, 21 nurses, 56 
shifts, 1176 nurses' working records per dataset, 50 datasets with total 58800 nurse assignment records). Table 5 shows the results of this test.

\begin{tabular}{llll}
\hline \multicolumn{4}{c}{ Test of Homogeneity of Variances } \\
\hline Levene Statistic & df1 & df2 & Sig. \\
5375.601 & 3 & 58796 & $.000 * * *$ \\
\hline
\end{tabular}

Table 5: Levene Test of Initial Results

For the approach variable, the $F$ value for Levene's test is $F(3,58796)=5375.601$, $(p=.000)$. We reject the null hypothesis of equality of variance $(p<.05)$ among four different policies, i.e., the assumption of homogeneity of variance is not met.

The p-value (.000) less than .05 in Table 5 indicates equal variance assumption is violated, hence we need to use an adjusted F-statistic. "The F test is fairly robust against inequality of variances if the sample sizes are equal, although the chance increases of incorrectly reporting a significant difference in the means when none exists" (Statguide, 1997). We use Welch ANOVA instead of a one-way ANOVA because the Welch test is more powerful and conservative than the Brown-Forsythe adjusted F statistic.

\begin{tabular}{lllll}
\hline \multicolumn{5}{c}{ Robust Tests of Equality of Means } \\
\hline Welch & Statistic $^{\mathrm{a}}$ & $\mathrm{df1}$ & $\mathrm{df2}$ & Sig. \\
Brown- & 9324.615 & 3 & 28728.049 & $.000^{* * *}$ \\
$\begin{array}{l}\text { Forsythe } \\
\text { a. Asymptotically F distributed. }\end{array}$ & 6123.720 & 3 & 40215.722 & $.000^{* * *}$ \\
\hline
\end{tabular}

\section{Table 6: Welch ANOVA Result of Initial Results}

Using the Welch statistic (shown in Table 6), we find that $\mathrm{F}(3,28728.049)=$ 9364.615, $p=.000$. We conclude that the adjusted $F$ ratio is significant $(p<.05)$. We reject the null hypothesis and proceed to compare the Post hoc approaches means. Post hoc comparisons using the Tukey HSD resulted in mean and standard deviation values for each 
method as follows: $\mathrm{WB}(\mathrm{M}=0.07, \mathrm{SD}=0.08) ; \mathrm{CC}-\mathrm{WB}(\mathrm{M}=0.17, \mathrm{SD}=0.16) ; \mathrm{CC}(\mathrm{M}=$ $0.37, \mathrm{SD}=0.29)$; and $\mathrm{NB}(\mathrm{M}=0.33, \mathrm{SD}=0.25)$. The test results indicate that each method produces output that differs significantly from all other methods $(p=.000, p<0.05$ for all method comparisons). The Tukey results demonstrate the differentiation of results, which allows us to proceed to consider the implications of these results.

We present the AAI and PCC results in Table 7 for our four methods of interest (WB, CC-WB, CC, and NB), as well as standard deviation for AAI for all methods. We consider the goal of acuity balance, continuity of care, and the tradeoffs between these goals. The AAI metric represents the sum of all acuity imbalances (difference from average for each nurse in a shift) across all shifts, we use this metric to demonstrate the accumulated effects in terms of workload imbalance over time.

We also provide a standard deviation of acuity imbalance for each method to demonstrate the relative stability of acuity imbalance provided by each method. This is not strictly the SD for AAI since we have only one value of AAI for each simulated data set (50 values). Rather, this SD value is calculated for the acuity imbalance per nurse per shift over a set of 50 datasets, each with 56 shifts. This gives us a much more robust sense of the acuity imbalance stability of each method.

\begin{tabular}{llll}
\hline Method & AAI & SD & PCC \\
\hline WB & 21.56 & 0.08 & $17.28 \%$ \\
CC-WB & 51.26 & 0.16 & $59.33 \%$ \\
CC & 108.23 & 0.29 & $58.83 \%$ \\
NB & 96.09 & 0.25 & $19.44 \%$ \\
\hline
\end{tabular}

Table 7: AAI and PCC Performance for Simple Heuristic Methods

As expected, the WB method clearly provides the best workload balance based on acuity, with the lowest AAI result of 21.56. The NB case, with AAI of 96.09, is a neutral 
case, representing neither quantitative nor qualitative goals, and therefore represents a natural counterpoint to the WB method, i.e., it defines a natural opposite "endpoint" to define a range of AAI values for our analysis. This provides an AAI range of 74.53 between the acuity-minimizing case (WB) and the NB case. The WB and Cen methods produced PCC results of $17.28 \%$ and $19.44 \%$, respectively. As expected, even without incorporating prior relationships into decision-making, these methods produced some assignments that match prior-care relationships.

The CC and CC-WB methods provide the highest PCC performance $(58.83 \%$ and $59.33 \%$ respectively $). \mathrm{CC}$ has the worst worse $\mathrm{AAI}(\mathrm{AAI}=108.23 ; \mathrm{PCC}=58.83 \%)$ and demonstrates the effect of maximizing prior-care assignments without incorporating a workload balance goal, clearly showing a tradeoff between the two performance goals. In fact, $\mathrm{CC}$ generates an AAI result that produces significantly greater acuity imbalance than an NB policy. Forcing continuity of care based on prior assignments further exacerbates the imbalance in workload distribution based on WB policy.

CC-WB enables similar PCC with a significant reduction in workload imbalance (AAI of 51.26). The tradeoff still exists. The CC-WB method provides a substantial increase in the relationship-based assignment (PCC of 59.33\% for CC-WB and $17.28 \%$ for WB), for a penalty/tradeoff in acuity imbalance (AAI of 51.26 for CC-WB and 21.56 for WB).

To explore the performance of the CC-WB heuristic, we calculate the usage of continuity of care. The average total potential continuity of care relationship is 1031.84 among all 50 datasets. The actual usage of continuity of care relationship is 944.50 among 
all 50 datasets, $91.54 \%$ of the potential usage. The remaining unused continuity of care contained $(8.46 \%)$ two parts: $44.56(4.32 \%)$ unused continuity of care relationship (multiple nurses cared one patient before, we could only choose one care relationship and unselected other existed relationships); 42.78 (4.14\%) discarded continuity of care relationship (one nurse cared one patient before, but based on N-P Ratio, workload balance among all nurses, these care relationship could not be used, and these patients have to be assigned based on workload).

To summarize the results, when compared with the WB method that showed the best performance in terms of acuity imbalance, our CC-WB method offered a tradeoff between workload balance (51.26 AAI of CC-WB policy versus 21.56 AAI of WB policy) and continuity of care (59.33\% PCC of CC-WB policy versus $17.28 \%$ PCC of WB policy). In the following section, we explore the benefits of using a more complex model to break the tradeoff.

\section{Acuity Threshold Policy}

In this section, we consider an improvement to the hybrid model, based on the use of an acuity threshold, relaxing the goal of maximizing continuity of care assignment. The acuity threshold $d$ provides a means of controlling the extent to which we apply the continuity-based assignment, targeting customers based on acuity to break the tradeoff between efficiency and service (Frei, 2006). We first explore the impact on AAI and PCC as we vary the acuity threshold $d$ from $d=0$ to $d=1.0$, where $d=0$ represents the CCWB case where the relationship-based assignment is maximized, and $d=1$ represents the WB case, where all patients are assigned to nurses based on minimizing accumulated acuity 
imbalance. By prioritizing higher-acuity patients for the relationship-based assignment we attempt to provide the benefits of continuity of care to those patients with the greatest needs since their tasks are more complex and they need more care than low acuity patients (Stanton and Stanton, 2004). We then analyze the sensitivity of results to cases where we vary key assumptions, namely the distribution of patient's acuity, shift length, the nursepatient, skill-matching requirements and the possibility of adding nurses from a resource pool to avoid overtime.

\section{Results for Acuity Threshold Policy}

The baseline experimental results show a clear tradeoff between AAI and PCC in Table 8. To alleviate such tradeoff, we consider the use of an acuity threshold. We vary the acuity threshold $d$ from $d=0$ to $d=1.0$, where $d=0$ represents the CC-WB case, and $d=1$ represents the WB case. Patient acuity scores vary between $[1.0 \ldots 0.0]$, so testing larger or smaller threshold values would be unlikely to provide further illumination. We change in the plot PCC for P-NCC (percentage of non-continuity of care, each equals 1-PCC) for ease of illustration of results, without loss of rigor. 


\begin{tabular}{llll}
\hline Threshold value & AAI & PCC & $\begin{array}{l}\text { P-NCC } \\
\text { (Plotting } \\
\text { purpose) }\end{array}$ \\
\hline $\mathrm{d}=1.0(\mathrm{WB})$ & 21.56 & $17.28 \%$ & $82.72 \%$ \\
$\mathrm{~d}=0.9$ & 21.65 & $17.95 \%$ & $82.05 \%$ \\
$\mathrm{~d}=0.8$ & 21.87 & $19.09 \%$ & $80.91 \%$ \\
$\mathrm{~d}=0.7$ & 22.16 & $20.71 \%$ & $79.29 \%$ \\
$\mathrm{~d}=0.6$ & 23.66 & $27.78 \%$ & $72.22 \%$ \\
$\mathrm{~d}=0.5$ & 27.94 & $38.74 \%$ & $61.26 \%$ \\
$\mathrm{~d}=0.4$ & 33.28 & $49.52 \%$ & $50.48 \%$ \\
$\mathrm{~d}=0.3$ & 38.98 & $55.77 \%$ & $44.23 \%$ \\
$\mathrm{~d}=0.2$ & 42.36 & $57.29 \%$ & $42.71 \%$ \\
$\mathrm{~d}=0.1$ & 47.72 & $58.53 \%$ & $41.47 \%$ \\
$\mathrm{~d}=0(\mathrm{CC}-\mathrm{WB})$ & 51.26 & $59.33 \%$ & $40.67 \%$ \\
\hline
\end{tabular}

Table 8: AAI and PCC Performance for Acuity Threshold Policy

As shown in Figure 2, the extreme cases of $d=0$ representing the CC-WB case offers the solution with the lowest P-NCC and highest AAI, whereas $d=1$ represents the WB case offer the lowest AAI with P-NCC being the highest. Variations of the acuity threshold move the solution along the tradeoff frontier with increments of d decreasing work imbalance but increasing the P-NCC. 


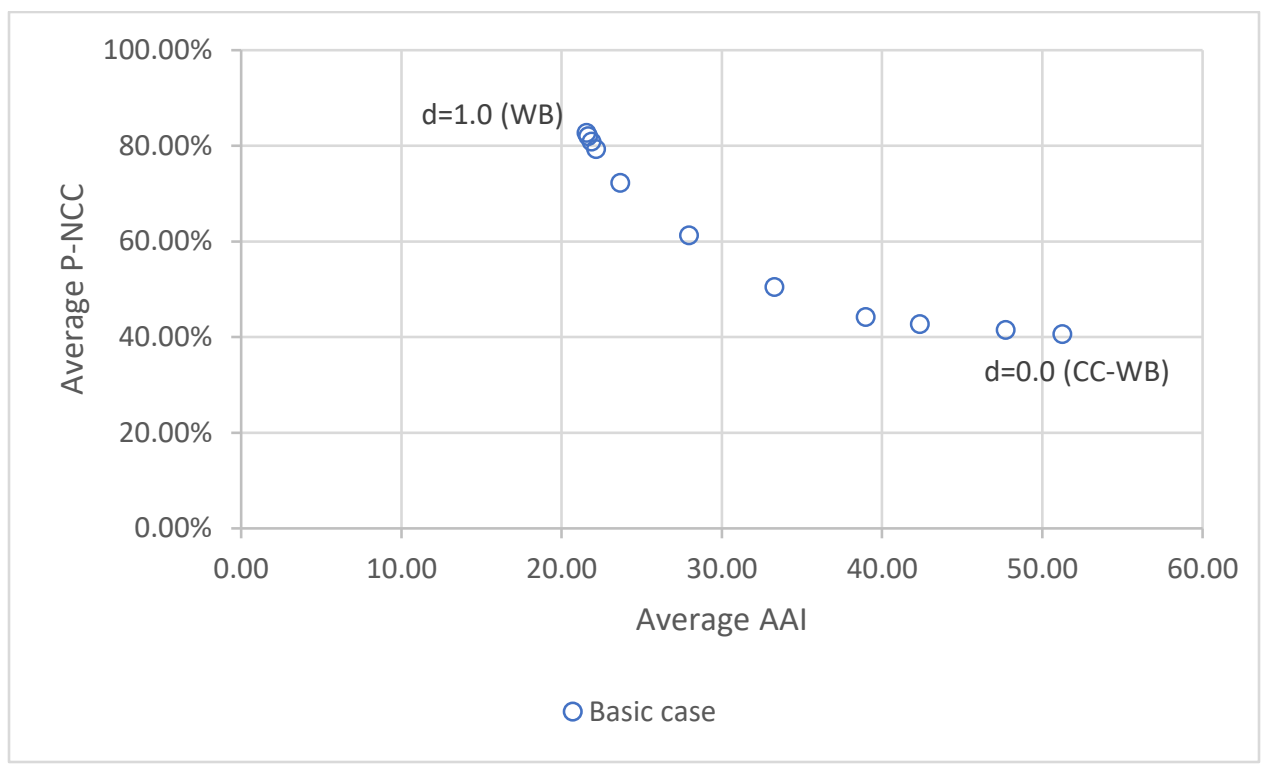

Figure 2: AAI Vs P-NCC for Baseline Case

We conduct a numerical analysis of the solution based on a preference model in which we provided weight 1 for the objective of minimizing AAI and weight 2 for the objective of minimizing P-NCC in Table 9. We then identify the "best solution" among all the thresholds with different combinations of preference weights for AAI and P-NCC. We provided detailed analytics in Table 9.

\begin{tabular}{|c|c|c|c|c|c|c|c|c|c|c|c|c|c|}
\hline & W1 for AAI & W2 for P-NCC & MIN OF $d=1$ & MIN OF d $=0.9$ & MIN OF $\mathrm{d}=0.8$ & MIN OF $d=0.7$ & $\mathrm{Fd}=0.6$ & MIN OF $d=0.5$ & MIN OF $d=0.4$ & MIN OF $d=0.3$ & MIN OF $d=0.2$ & MIN OF $d=0.1$ & $\mathrm{Fd}=0$ \\
\hline AAI & & & 21.56 & 21.65 & 21.87 & 22.16 & 23.66 & 27.94 & 33.28 & 38.98 & 42.36 & 47.72 & 51.26 \\
\hline \multirow{20}{*}{ P-NCC } & & & 82.72 & 82.05 & 80.91 & 79.29 & 72.22 & 61.26 & 50.48 & 44.23 & 42.71 & 41.47 & 40.67 \\
\hline & 0 & 1 & 82.72 & 82.05 & 80.91 & 79.29 & 72.22 & 61.26 & 50.48 & 44.23 & 42.71 & 41.47 & 40.67 \\
\hline & 0.1 & 0.9 & 76.60 & 76.01 & 75.01 & 73.58 & 67.36 & 57.93 & 48.76 & 43.71 & 42.68 & 42.10 & 41.73 \\
\hline & 0.15 & 0.85 & 73.55 & 72.99 & 72.05 & 70.72 & 64.94 & 56.26 & 47.90 & 43.44 & 42.66 & 42.41 & 42.26 \\
\hline & 0.2 & 0.8 & 70.49 & 69.97 & 69.10 & 67.86 & 62.51 & 54.60 & 47.04 & 43.18 & 42.64 & 42.72 & 42.79 \\
\hline & 0.25 & 0.75 & 67.43 & 66.95 & 66.15 & 65.01 & 60.08 & 52.93 & 46.18 & 42.92 & 42.62 & 43.03 & 43.32 \\
\hline & 0.3 & 0.7 & 64.37 & 63.93 & 63.20 & 62.15 & 57.65 & 51.26 & 45.32 & 42.66 & 42.61 & 43.35 & 43.85 \\
\hline & 0.35 & 0.65 & 61.31 & 60.91 & 60.25 & 59.29 & 55.22 & 49.60 & 44.46 & 42.39 & 42.59 & 43.66 & 44.38 \\
\hline & 0.4 & 0.6 & 58.26 & 57.89 & 57.29 & 56.44 & 52.80 & 47.93 & 43.60 & 42.13 & 42.57 & 43.97 & 44.91 \\
\hline & 0.45 & 0.55 & 55.20 & 54.87 & 54.34 & 53.58 & 50.37 & 46.27 & 42.74 & 41.87 & 42.55 & 44.28 & 45.44 \\
\hline & 0.5 & 0.5 & 52.14 & 51.85 & 51.39 & 50.73 & 47.94 & 44.60 & 41.88 & 41.61 & 42.54 & 44.60 & 45.97 \\
\hline & 0.55 & 0.45 & 49.08 & 48.83 & 48.44 & 47.87 & 45.51 & 42.93 & 41.02 & 41.34 & 42.52 & 44.91 & 46.49 \\
\hline & 0.6 & 0.4 & 46.02 & 45.81 & 45.49 & 45.01 & 43.08 & 41.27 & 40.16 & 41.08 & 42.50 & 45.22 & 47.02 \\
\hline & 0.65 & 0.35 & 42.97 & 42.79 & 42.53 & 42.16 & 40.66 & 39.60 & 39.30 & 40.82 & 42.48 & 45.53 & 47.55 \\
\hline & 0.7 & 0.3 & 39.91 & 39.77 & 39.58 & 39.30 & 38.23 & 37.94 & 38.44 & 40.56 & 42.47 & 45.85 & 48.08 \\
\hline & 0.75 & 0.25 & 36.85 & 36.75 & 36.63 & 36.44 & 35.80 & 36.27 & 37.58 & 40.29 & 42.45 & 46.16 & 48.61 \\
\hline & 0.8 & 0.2 & 33.79 & 33.73 & 33.68 & 33.59 & 33.37 & 34.60 & 36.72 & 40.03 & 42.43 & 46.47 & 49.14 \\
\hline & 0.85 & 0.15 & 30.73 & 30.71 & 30.73 & 30.73 & 30.94 & 32.94 & 35.86 & 39.77 & 42.41 & 46.78 & 49.67 \\
\hline & 0.9 & 0.1 & 27.68 & 27.69 & 27.77 & 27.87 & 28.52 & 31.27 & 35.00 & 39.51 & 42.40 & 47.10 & 50.20 \\
\hline & 1 & 0 & 21.56 & 21.65 & 21.87 & 22.16 & 23.66 & 27.94 & 33.28 & 38.98 & 42.36 & 47.72 & 51.26 \\
\hline
\end{tabular}

Table 9: Preference Model for Baseline Case 
We transfer the preferences table (Table 9) into the threshold figure (Figure 3) by connecting all suggest preferences point of each weight pair. Here, we first provide different weights for AAI (W1) and P-NCC (W2) pair. We then calculate the preferences use AAI*W1 + P-NCC*W2. We capture the lowest points of (AAI+P-NCC) for each weight pair which represents the preferred points.

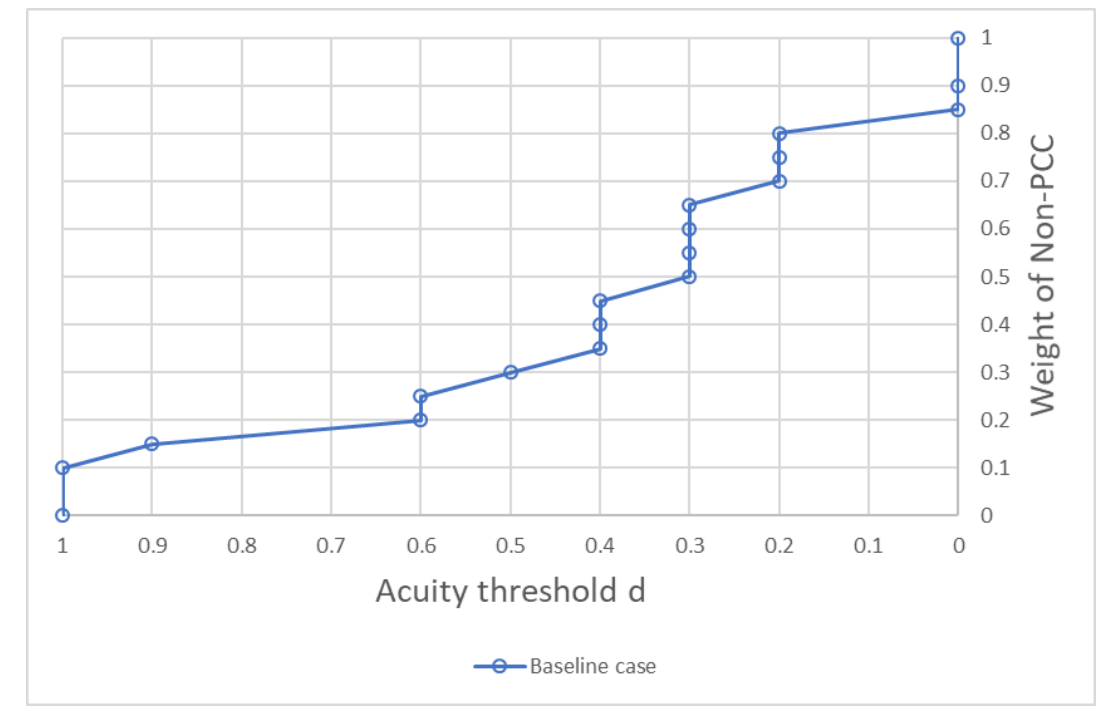

Figure 3: Best Acuity Threshold based on Preference Weight

Figure 3 is a simplification of Table 9, in which we transfer the green points of the preference table into a curve. The boxed area in the top part of Figure 4 [A] shows P-NCC from 1.0 to 0.5 . This shows preferences in the context of an already strong preference for continuity, which is not what we are interested in. 

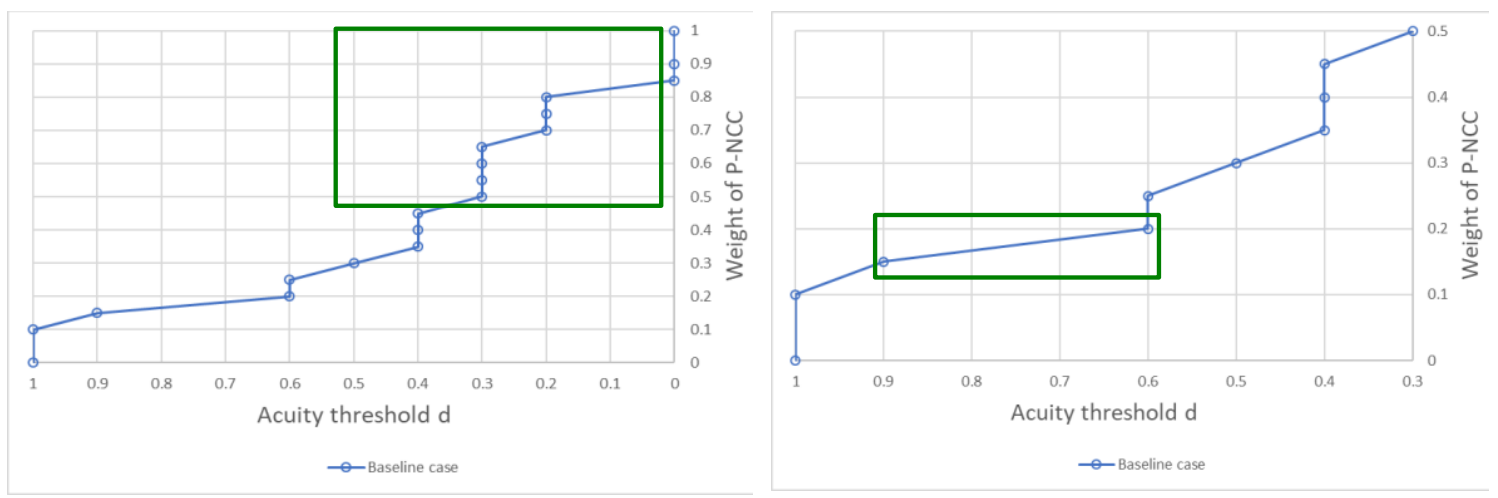

Figure 4: [A] High Weight Area for P-NCC; [B] Example Horizontal Run

In Figure 4 [B], we show only the bottom half of the plot, up to P-NCC 0.5. This is the area of our interest, where we can look at preferences for high weight on AAI. In these range of weights, we want to choose a value for $d$ to represent both minimizations of both workload imbalance and percentage of non-continuity of care. That is, the suggested threshold should be close to 0 in $\mathrm{Y}$-axis for greater weight on AAI, and the value of $d$ should be as low as possible to maximize the number of patients assigned based on care relationships. To achieve these two goals, we want to have the range of the curve with a large variation on the horizontal axis and little change on the vertical axis. Based on Figure 4 [B], we concentrate on the curve from $(d=0.9$ to $d=0.6)$. At the low end of this range where $d=0.6$, we get many patients involved in the continuity of care for little increase of workload imbalance (captured in the lower preference point number) at a very high weight of AAI preference.

\section{Model Sensitivity Analysis Results}

Following the main experimental evaluation, we conducted additional studies to understand the sensitivity of our acuity-threshold model to key assumptions related to 
organizational and contextual factors that may vary across hospital units. We consider five different cases, the distribution of patient's acuity level, the nurse shift length, the nurse-to-patient ratio, patients with special care needs, and hard constraints on the nursepatient ratio while allowing temporary nurses to be drawn from a nurse pool.

The summary of our five different cases are shown below in Table 10:

\section{Environment factors}

Case1: Acuity distribution change

1. Left skewed $15 \%$ observation unit

2. Normal distribution general medical unit (baseline case)

3. Right skewed $15 \%$ post-surgery unit

Case 2: Nurse schedule change

1. 12 hours shift 56 shifts in 4 weeks (baseline case)

2. 8 hours shift 84 shifts in 4 weeks

Case 3: N-P Ratio change

1. 1 nurse care 3 patients per shift

2. 1 nurse care 6 patients per shift (baseline case)

3. 1 nurse care 9 patients per shift

Case 4: Patient's with special requirement

1. No special requirement (baseline case)

2. $20 \%$ patient with requirement, $40 \%$ nurse with skill

Case 5: Add nurses from pool if N-P Ratio exceed 6

1. Additional nurses added from pool with some shifts

2. Normal distribution general medical unit (baseline case)

\section{Table 10: Summary of 5 Different Environment Cases}




\section{Case1: Acuity Distribution Change}

\section{Left Skewed Case}

We shift the patients' acuity from normal distribution general inpatient unit to left skewed distribution around $15 \%$ (e.g., Obstetrics unit) with a mean of 0.35 (acuity scores fall in the range $[0 \ldots 1.0])$.

The average total potential continuity of care relationship is 1032.50 among all 50 datasets. The actual usage of continuity of care relationship is 944.42 among all 50 datasets. We use the actual usage divided by the total potential to get the usage of continuity of care. The actual usage of continuity of care among 50 datasets is $91.46 \%$. The remaining unused continuity of care contained (8.53\%) two parts: $44.56(4.32 \%)$ unused continuity of care relationship (multiple nurses cared one patient before, we could only choose one care relationship and unselected other existed relationships); 43.52 (4.22\%) discarded continuity of care relationship (one nurse cared one patient before, but based on N-P Ratio, workload balance among all nurses, these care relationship could not be used, and these patients have to be assigned based on workload). 


\begin{tabular}{|c|c|c|}
\hline Method & AAI & P-NCC \\
\hline $\mathrm{d}=1.0(\mathrm{WB})$ & 13.97 & $82.70 \%$ \\
\hline $\mathrm{d}=0.9$ & 13.97 & $82.66 \%$ \\
\hline $\mathrm{d}=0.8$ & 13.98 & $82.23 \%$ \\
\hline $\mathrm{d}=0.7$ & 14.57 & $81.44 \%$ \\
\hline $\mathrm{d}=0.6$ & 15.73 & $79.73 \%$ \\
\hline $\mathrm{d}=0.5$ & 17.82 & $77.19 \%$ \\
\hline $\mathrm{d}=0.4$ & 21.72 & $68.67 \%$ \\
\hline $\mathrm{d}=0.3$ & 29.86 & $54.04 \%$ \\
\hline $\mathrm{d}=0.2$ & 36.09 & $45.23 \%$ \\
\hline $\mathrm{d}=0.1$ & 41.90 & $42.09 \%$ \\
\hline $\mathrm{d}=0(\mathrm{CC}-\mathrm{WB})$ & 45.79 & $40.73 \%$ \\
\hline
\end{tabular}

Table 11: AAI Vs P-NCC for Left 15\%

Based on Table 11, the whole trend of AAI vs P-NCC for left 15\% case move to left compares to the baseline case, which means lower AAI range along with similar PNCC. We conduct Table 11 to a numerical analysis of the solution (Table 12) based on a preference model in which we provided weight 1 for the objective of minimizing AAI and weight 2 for the objective of minimizing P-NCC.

\begin{tabular}{|c|c|c|c|c|c|c|c|c|c|c|c|c|c|}
\hline & W1 for AAI & W2 for P-NCC & MIN OF $d=1$ & MIN OF d $=0.9$ & MIN OF $d=0.8$ & MIN OF d $=0.7$ & MIN OF $d=0.6$ & MIN OF d $=0.5$ & MIN OF d $=0.4$ & MIN OF $d=0.3$ & MIN OF d $d=0.2$ & MIN OF $d=0.1$ & MIN OF $d=0$ \\
\hline AAI & & & 13.97 & 13.97 & 13.98 & 14.57 & 15.73 & 17.82 & 21.72 & 29.86 & 36.09 & 41.90 & 45.79 \\
\hline \multirow[t]{20}{*}{ P-NCC } & & & 82.70 & 82.66 & 82.23 & 81.44 & 79.73 & 77.19 & 68.67 & 54.04 & 45.23 & 42.09 & 40.73 \\
\hline & 0 & 1 & 82.70 & 82.66 & 82.23 & 81.44 & 79.73 & 77.19 & 68.67 & 54.04 & 45.23 & 42.09 & 40.73 \\
\hline & 0.1 & 0.9 & 75.83 & 75.80 & 75.41 & 74.75 & 73.33 & 71.25 & 63.97 & 51.62 & 44.32 & 42.07 & 41.23 \\
\hline & 0.15 & 0.85 & 72.39 & 72.36 & 71.99 & 71.41 & 70.13 & 68.28 & 61.62 & 50.41 & 43.86 & 42.06 & 41.49 \\
\hline & 0.2 & 0.8 & 68.95 & 68.93 & 68.58 & 68.07 & 66.93 & 65.31 & 59.28 & 49.20 & 43.40 & 42.05 & 41.74 \\
\hline & 0.25 & 0.75 & 65.52 & 65.49 & 65.17 & 64.72 & 63.73 & 62.35 & 56.93 & 47.99 & 42.95 & 42.04 & 41.99 \\
\hline & 0.3 & 0.7 & 62.08 & 62.06 & 61.76 & 61.38 & 60.53 & 59.38 & 54.58 & 46.78 & 42.49 & 42.03 & 42.24 \\
\hline & 0.35 & 0.65 & 58.64 & 58.62 & 58.34 & 58.04 & 57.33 & 56.41 & 52.24 & 45.58 & 42.03 & 42.02 & 42.50 \\
\hline & 0.4 & 0.6 & 55.21 & 55.19 & 54.93 & 54.69 & 54.13 & 53.44 & 49.89 & 44.37 & 41.58 & 42.01 & 42.75 \\
\hline & 0.45 & 0.55 & 51.77 & 51.75 & 51.52 & 51.35 & 50.93 & 50.47 & 47.54 & 43.16 & 41.12 & 42.00 & 43.00 \\
\hline & 0.5 & 0.5 & 48.33 & 48.32 & 48.11 & 48.01 & 47.73 & 47.50 & 45.19 & 41.95 & 40.66 & 41.99 & 43.26 \\
\hline & 0.55 & 0.45 & 44.90 & 44.88 & 44.69 & 44.66 & 44.53 & 44.54 & 42.85 & 40.74 & 40.21 & 41.98 & 43.51 \\
\hline & 0.6 & 0.4 & 41.46 & 41.45 & 41.28 & 41.32 & 41.33 & 41.57 & 40.50 & 39.53 & 39.75 & 41.97 & 43.76 \\
\hline & 0.65 & 0.35 & 38.02 & 38.02 & 37.87 & 37.98 & 38.13 & 38.60 & 38.15 & 38.32 & 39.29 & 41.96 & 44.02 \\
\hline & 0.7 & 0.3 & 34.59 & 34.58 & 34.46 & 34.63 & 34.93 & 35.63 & 35.81 & 37.12 & 38.83 & 41.95 & 44.27 \\
\hline & 0.75 & 0.25 & 31.15 & 31.15 & 31.04 & 31.29 & 31.73 & 32.66 & 33.46 & 35.91 & 38.38 & 41.95 & 44.52 \\
\hline & 0.8 & 0.2 & 27.71 & 27.71 & 27.63 & 27.95 & 28.53 & 29.69 & 31.11 & 34.70 & 37.92 & 41.94 & 44.77 \\
\hline & 0.85 & 0.15 & 24.28 & 24.28 & 24.22 & 24.60 & 25.33 & 26.73 & 28.76 & 33.49 & 37.46 & 41.93 & 45.03 \\
\hline & 0.9 & 0.1 & 20.84 & 20.84 & 20.81 & 21.26 & 22.13 & 23.76 & 26.42 & 32.28 & 37.01 & 41.92 & 45.28 \\
\hline & 1 & 0 & 13.97 & 13.97 & 13.98 & 14.57 & 15.73 & 17.82 & 21.72 & 29.86 & 36.09 & 41.90 & 45.79 \\
\hline
\end{tabular}

\section{Table 12: Preference Model for Left 15\% Case}

From Table 12, we could see two major different preference changes over time. When we put a preference for AAI (1.0) and preference for P-NCC (0.0), the "best solution" for $d$ is 1.0. When we put a preference for AAI (from 0.9 to 0.65 ) and preference 
for P-NCC (from 0.1 to 0.35 ), the "best solution" for $d$ is 0.8 and skipped solution in 0.9 . When we put a preference for AAI (from 0.6 to 0.0 ) and preference for P-NCC (from 0.4 to 1.0 ), the range of "best solution" for $d$ is 0.3 to 1.0 and skipped solutions from 0.7 to 0.4 .

Since patients' acuities are more left skewed which means most patients have lower acuity scores and could be assigned easier in each shift. Therefore, the WB function has an important impact on the assignment ( 6 "best solution" concentered on $d=0.8$ ). The CC function begins to play an important role while "most" weight preferences are put on PNCC since the natural patients' acuity setting is easy to be fairly assigned (11 'best solution" equally distributed from $d=0.6$ to 0.0 ). Compared to the basic (normal distribution) case, the range of "best solution" for Left 15\% Case is narrower (i.e., 19 equally distributed from $d=1.0$ to $d=0.0$ with $0.8,0.7$, and 0.1 skipped for baseline case vs more left skewed, i.e., 6 concentered on 0.8 and right skewed i.e., 12 concentered from 0.3 to 0.0 with $0.9,0.7,0.6,0.5$, and 0.4 skipped.

\section{Right Skewed Case}

We shift patients' acuity from normal distribution general inpatient unit to right skewed distribution around 15\% (e.g., Medical-Surgical unit) with a mean of 0.64 (acuity scores fall in the range $[0 \ldots 1.0])$.

The average total potential continuity of care relationship is 1031.38 among all 50 datasets. The actual usage of continuity of care relationship is 944.58 among all 50 datasets. We use the actual usage divided by the total potential to get the usage of continuity of care. The actual usage of continuity of care among 50 datasets is $91.58 \%$. 
The remaining unused continuity of care contained (8.42\%) two parts: 44.04 (4.27\%) unused continuity of care relationship (multiple nurses cared one patient before, we could only choose one care relationship and unselected other existed relationships); 42.76 (4.15\%) discarded continuity of care relationship (one nurse cared one patient before, but based on N-P Ratio, workload balance among all nurses, these care relationship could not be used, and these patients have to be assigned based on workload).

\begin{tabular}{llc}
\hline Method & AAI & P-NCC \\
\hline$d=1.0(W B)$ & 33.37 & $82.76 \%$ \\
$d=0.9$ & 33.17 & $80.97 \%$ \\
$d=0.8$ & 32.55 & $77.78 \%$ \\
$d=0.7$ & 31.78 & $68.53 \%$ \\
$d=0.6$ & 32.34 & $54.05 \%$ \\
$d=0.5$ & 33.16 & $45.75 \%$ \\
$d=0.4$ & 34.83 & $43.58 \%$ \\
$d=0.3$ & 38.81 & $42.13 \%$ \\
$d=0.2$ & 43.91 & $41.20 \%$ \\
$d=0.1$ & 46.53 & $40.69 \%$ \\
$d=0($ CC-WB $)$ & 46.53 & $40.69 \%$ \\
Table 13: AAI Vs P-NCC for Right 15\%
\end{tabular}

Based on Table 13, the whole trend of AAI vs P-NCC for right $15 \%$ case move to right compare to the baseline case, which means higher AAI range along with similar PNCC. We conduct to a numerical analysis of the solution based on a preference model in Table 14, which we provided weight 1 for the objective of minimizing AAI and weight 2 for the objective of minimizing P-NCC. 


\begin{tabular}{|c|c|c|c|c|c|c|c|c|c|c|c|c|c|}
\hline & W1 for AAI & W2 for P-NCC & MIN OF $d=1$ & MIN OF $d=0.9$ & MIN OF $d=0.8$ & MIN OF d=0.7 & MIN OF $d=0.6$ & MIN OF $d=0.5$ & MIN OF $d=0.4$ & MIN OF $d=0.3$ & MIN OF $d=0.2$ & MIN OF $d=0.1$ & MIN OF $d=0$ \\
\hline AAI & & & 33.37 & 33.17 & 32.55 & 31.78 & 32.34 & 33.16 & 34.83 & 38.81 & 43.91 & 46.53 & 46.53 \\
\hline \multirow[t]{20}{*}{ P-NCC } & & & 82.76 & 80.97 & 77.78 & 68.53 & 54.05 & 45.75 & 43.58 & 42.13 & 41.20 & 40.69 & 40.69 \\
\hline & 0 & 1 & 82.76 & 80.97 & 77.78 & 68.53 & 54.05 & 45.75 & 43.58 & 42.13 & 41.20 & 40.69 & 40.69 \\
\hline & 0.1 & 0.9 & 77.82 & 76.19 & 73.26 & 64.86 & 51.88 & 44.49 & 42.71 & 41.80 & 41.47 & 41.27 & 41.27 \\
\hline & 0.15 & 0.85 & 75.35 & 73.80 & 71.00 & 63.02 & 50.79 & 43.86 & 42.27 & 41.63 & 41.60 & 41.56 & 41.56 \\
\hline & 0.2 & 0.8 & 72.88 & 71.41 & 68.73 & 61.18 & 49.71 & 43.23 & 41.83 & 41.47 & 41.74 & 41.85 & 41.85 \\
\hline & 0.25 & 0.75 & 70.41 & 69.02 & 66.47 & 59.34 & 48.62 & 42.60 & 41.39 & 41.30 & 41.88 & 42.15 & 42.15 \\
\hline & 0.3 & 0.7 & 67.94 & 66.63 & 64.21 & 57.51 & 47.54 & 41.97 & 40.96 & 41.14 & 42.01 & 42.44 & 42.44 \\
\hline & 0.35 & 0.65 & 65.47 & 64.24 & 61.95 & 55.67 & 46.45 & 41.34 & 40.52 & 40.97 & 42.15 & 42.73 & 42.73 \\
\hline & 0.4 & 0.6 & 63.00 & 61.85 & 59.69 & 53.83 & 45.36 & 40.71 & 40.08 & 40.80 & 42.28 & 43.02 & 43.02 \\
\hline & 0.45 & 0.55 & 60.53 & 59.46 & 57.43 & 51.99 & 44.28 & 40.08 & 39.64 & 40.64 & 42.42 & 43.32 & 43.32 \\
\hline & 0.5 & 0.5 & 58.06 & 57.07 & 55.16 & 50.16 & 43.19 & 39.45 & 39.20 & 40.47 & 42.55 & 43.61 & 43.61 \\
\hline & 0.55 & 0.45 & 55.59 & 54.68 & 52.90 & 48.32 & 42.11 & 38.83 & 38.77 & 40.31 & 42.69 & 43.90 & 43.90 \\
\hline & 0.6 & 0.4 & 53.12 & 52.29 & 50.64 & 46.48 & 41.02 & 38.20 & 38.33 & 40.14 & 42.83 & 44.19 & 44.19 \\
\hline & 0.65 & 0.35 & 50.65 & 49.90 & 48.38 & 44.64 & 39.94 & 37.57 & 37.89 & 39.98 & 42.96 & 44.48 & 44.48 \\
\hline & 0.7 & 0.3 & 48.19 & 47.51 & 46.12 & 42.81 & 38.85 & 36.94 & 37.45 & 39.81 & 43.10 & 44.78 & 44.78 \\
\hline & 0.75 & 0.25 & 45.72 & 45.12 & 43.86 & 40.97 & 37.77 & 36.31 & 37.02 & 39.64 & 43.23 & 45.07 & 45.07 \\
\hline & 0.8 & 0.2 & 43.25 & 42.73 & 41.59 & 39.13 & 36.68 & 35.68 & 36.58 & 39.48 & 43.37 & 45.36 & 45.36 \\
\hline & 0.85 & 0.15 & 40.78 & 40.34 & 39.33 & 37.29 & 35.59 & 35.05 & 36.14 & 39.31 & 43.51 & 45.65 & 45.65 \\
\hline & 0.9 & 0.1 & 38.31 & 37.95 & 37.07 & 35.46 & 34.51 & 34.42 & 35.70 & 39.15 & 43.64 & 45.94 & 45.94 \\
\hline & 1 & 0 & 33.37 & 33.17 & 32.55 & 31.78 & 32.34 & 33.16 & 34.83 & 38.81 & 43.91 & 46.53 & 46.53 \\
\hline
\end{tabular}

Table 14: Preference Model for Right 15\% Case

\section{Summary of Distribution Cases}

We report results where we shift patient's acuity from normal distribution general inpatient unit to left and right skewed distribution. Figure 5 is a simplification of Table 12 and Table 14, in which we transfer the green points of the preference table into a curve. Based on the similar logic in Figure 4 [B], we show only the bottom half of the plot, up to P-NCC 0.5 in Figure 5. Compared with the baseline case (normal distribution), the left-skewed solution moved to the left, which means lower AAI range along with similar P-NCC. The right-skewed solution shows an interesting result, which is from $d=0.7$ to $d=0.5$ we get increments of PCC without a significant penalty of AAI because most of the patients are high acuity and the initial AAI is high. 


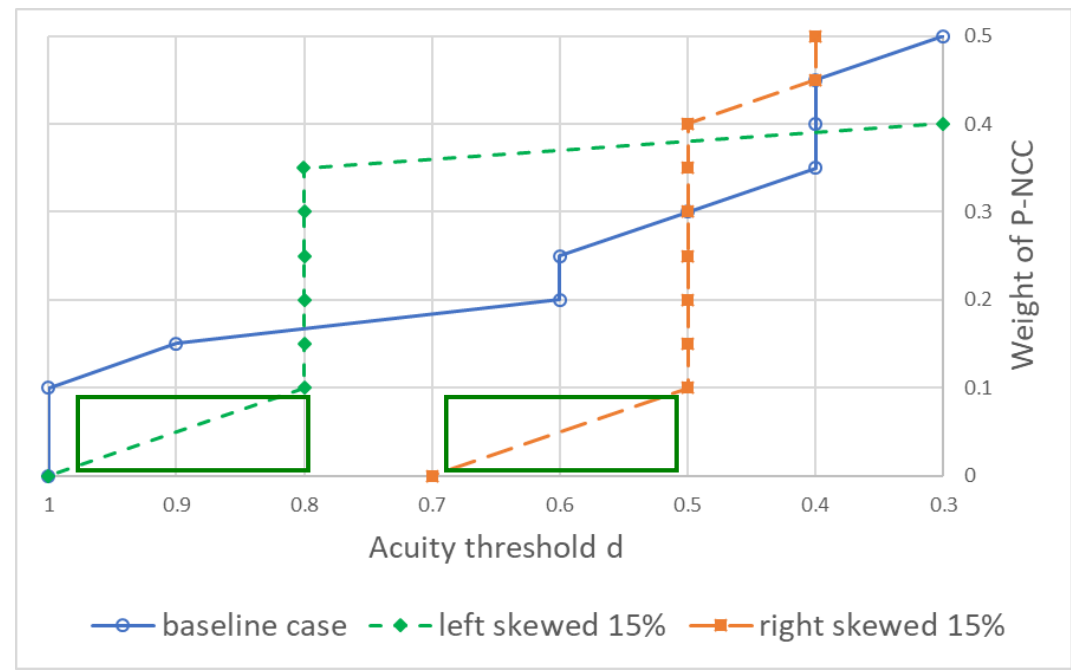

Figure 5: Example Horizontal Run of Case 1

In Figure 5, we concentrate on the curve from ( $d=1.0$ to $d=0.8)$ for the left skewed 15\% case. Since patients' acuities are more left skewed (which means most patients have lower acuity score), they are easier to be assigned based on acuity balance in each shift. Therefore, the weight of AAI has an important impact on the assignment (concentrated on $d=1.0$ to $d=0.8$ ). At the low end of this range where $d=0.8$, we get many patients involved in the continuity of care for little increase of workload imbalance (captured in the lower preference point number) at a very high weight of AAI preference.

Different from the left skewed case, we concentrate on the curve from ( $d=0.7$ to $d=0.5$ ) for the right skewed $15 \%$ case in Figure 5. Since patients' acuities are right skewed, which means most patients have high acuity score, we tend to have higher AAI imbalance compared to the baseline case (i.e., the start point of the right-skewed curve is 0.7 compare to the 0.9 of the baseline and 1.0 of left skewed curves). Hence, it is advantageous to start at a lower threshold, but requires a greater preference for P-NCC to 
shift the threshold. At the low end of this range where $d=0.5$, we get many patients involved in the continuity of care for little increase of workload imbalance (captured in the lower preference point number) at a very high weight of AAI preference.

\section{Case 2: Nurse Schedule Change}

Secondly, we consider variations in the length of the nurse-shift going from 12-hour to 8hour shift (three shifts per day). In this model, the patient's length of stay is

proportionally adjusted, and the patient's acuity is generated from the normal distribution over the larger number of shifts. The nurse allocated to shifts is also adjusted in proportion to the shift length; nurses still work for 40 hours per week and do not work multiple shifts in a row.

The average total potential continuity of care relationship is 2035.96 among all 50 datasets. The actual usage of continuity of care relationship is 1475.06 among all 50 datasets. We use the actual usage divided by the total potential to get the usage of continuity of care. The actual usage of continuity of care among 50 datasets is $72.45 \%$. The remaining unused continuity of care contained (27.55\%) two parts: $500.88(24.60 \%)$ unused continuity of care relationship (multiple nurses cared one patient before, we could only choose one care relationship and unselected other existed relationships) (in this case, nurse will have much higher percentage to meet the same patients they used to care before, and there are lots of cases two or more nurses have care relationship with patients in current shift since patients remain 180 for 8 hours shift in 1 month, patients length of stay increased to range 6 shifts to 30 shifts distributed normally around a mean of 12.09 , nurse work $33 \%$ more shifts per week (from 3 shifts to 5 shifts per week), and total shifts 
changed from 54 to 81 (shifts changed from 12 hours to 8 hours, $60.02(2.95 \%)$

discarded continuity of care relationship (one nurse cared one patient before, but based on N-P Ratio, workload balance among all nurses, these care relationship could not be used, and these patients have to be assigned based on workload).

\begin{tabular}{lll}
\hline Method & AAI & P-NCC \\
\hline$d=1.0(W B)$ & 31.84 & $76.17 \%$ \\
d=0.9 & 31.97 & $75.31 \%$ \\
d=0.8 & 32.41 & $74.38 \%$ \\
d=0.7 & 32.45 & $72.95 \%$ \\
d=0.6 & 34.20 & $66.94 \%$ \\
d $=0.5$ & 40.29 & $57.36 \%$ \\
d=0.4 & 48.53 & $47.91 \%$ \\
d=0.3 & 58.52 & $42.49 \%$ \\
$d=0.2$ & 64.86 & $41.04 \%$ \\
$d=0.1$ & 70.57 & $39.96 \%$ \\
$d=0(C C-W B)$ & 77.02 & $39.33 \%$ \\
\hline
\end{tabular}

Table 15: AAI Vs P-NCC for 8 hours shift

Based on Table 15, the whole trend of AAI vs P-NCC for 8 hours shift case does not change significantly compared to the baseline case. The performance in terms of PNCC does not change significantly, while nurses will have much higher percentage to meet the same patients they used to care before, there are many cases two or more nurses have care relationship with patients in the current shift and the usage of potential relationships is lower. Yet the AAI becomes higher, which represents greater work imbalance, because nurses work more frequently, and we are adding more shifts during the simulation period (4 weeks). The overall performance of 8 hours shift case shows a little higher AAI range along with similar P-NCC. We did not provide the table of preference here since the overall performance between baseline case and 8 hours shift is not significantly different. We only provided Figure 6 here to show how 8 hours curve is similar to the baseline curve. 


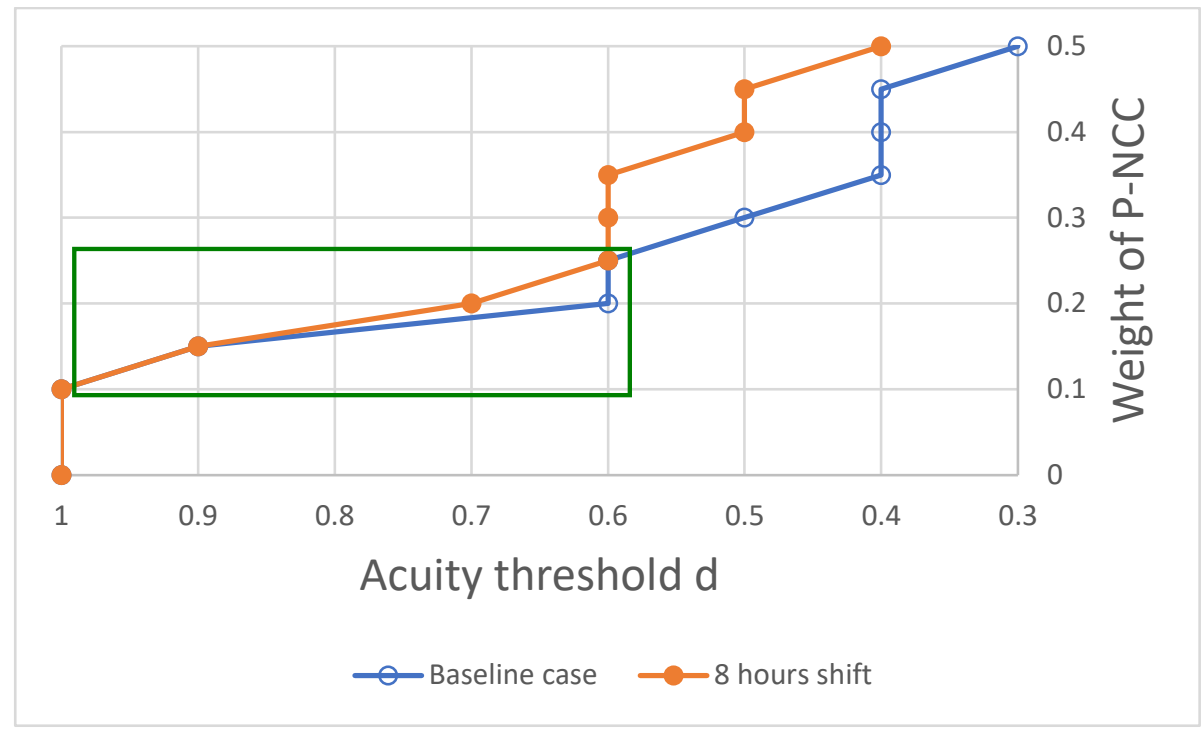

Figure 6: Example Horizontal Run of Case 2

Similar to the baseline case, the concentrated choices of 8 hours shift case are shown from $d=0.9$ until $d=0.6$ in Figure 6. Compare to the baseline case, the 8 hours shift creates more shifts for nurses to care patients, which did not impact the assignment results for workload balance. Therefore, the results of the 8 hours shift did not show significantly different compared to the baseline case.

Case 3: N-P Ratio change

Our base model assumes an N-P Ratio of 6 patients per nurse per shift. We now allow NP Ratio to vary, with both higher patient loads (1:9) and lower loads (1:3). When N-P Ratio equals 3 , the patient number will remain 180 but the number of nurses will increase from 21 to 42 in order to reach one nurse cares 3 patients. Most nurses work consecutive 3 days (41.24\%) or 4 days (40.21\%) per week, due to the complexity of scheduling the percentage of nurses working isolated shifts increased. Similarly, when N-P Ratio is 9 patients per nurse per shift, the number of nurses will decrease from 21 to 14 in order to 
reach one nurse cares 9 patients. Still, as in the baseline case, each nurse will work 14 days per month in each case.

N-P Ratio changes from 6 to 3

We shift N-P Ratio 6 patients per nurse per shift to 3 patients per nurse per shift with normal distribution with a mean of 0.50 (acuity scores fall in the range $[0 \ldots 1.0]$ ). Most nurses work consecutive 3 days (41.24\%) or 4 days $(40.21 \%)$ per week, fewer nurses $(18.56 \%)$ (due to the complexity of schedule, nurse work isolated shifts increased) work isolated days on or day off, each nurse will work 14 days per month which fit the real nurse scheduling (Azaiez and Al Sharif, 2005).

The average total potential continuity of care relationship is 1056.46 among all 50 datasets. The actual usage of continuity of care relationship is 873.20 among all 50 datasets. We use the actual usage divided by the total potential to get the usage of continuity of care. The actual usage of continuity of care among 50 datasets is $82.65 \%$. The remaining unused continuity of care contained (17.35\%) two parts: $84.08(7.96 \%)$ unused continuity of care relationship (multiple nurses cared one patient before, we could only choose one care relationship and unselected other existed relationships); 99.18 (9.39\%) discarded continuity of care relationship (one nurse cared one patient before, but based on N-P Ratio, workload balance among all nurses, these care relationship could not be used, and these patients have to be assigned based on workload). 


\begin{tabular}{lll}
\hline Method & AAI & P-NCC \\
\hline$d=1.0($ WB $)$ & 31.84 & $76.17 \%$ \\
d $=0.9$ & 31.97 & $75.31 \%$ \\
d=0.8 & 32.41 & $74.38 \%$ \\
d $=0.7$ & 32.45 & $72.95 \%$ \\
d $=0.6$ & 34.20 & $66.94 \%$ \\
$d=0.5$ & 40.29 & $57.36 \%$ \\
$d=0.4$ & 48.53 & $47.91 \%$ \\
$d=0.3$ & 58.52 & $42.49 \%$ \\
$d=0.2$ & 64.86 & $41.04 \%$ \\
$d=0.1$ & 70.57 & $39.96 \%$ \\
$d=0($ CC-WB $)$ & 77.02 & $39.33 \%$ \\
\hline
\end{tabular}

\section{Table 16: AAI Vs P-NCC for N-P Ratio 3}

Based on Table 16, the whole trend of AAI vs P-NCC for N-P Ratio 3 case move to right compare to the baseline case, which means higher AAI range along with similar PNCC. We conduct to a numerical analysis of the solution based on a preference model in Table 17, which we provided weight 1 for the objective of minimizing AAI and weight 2 for the objective of minimizing P-NCC.

\begin{tabular}{|c|c|c|c|c|c|c|c|c|c|c|c|c|c|}
\hline & W1 for AAI & W2 for P-NCC & MIN OF $d=1$ & MIN OF d=0.9 & MIN OF $\mathrm{d}=0.8$ & MIN OF $d=0.7$ & MIN OF $d=0.6 \mathrm{~N}$ & MIN OF $d=0.5$ & MIN OF $d=0.4$ & MIN OF $d=0.3$ & MIN OF $d=0.2$ & MIN OF $d=0.1 \mathrm{~N}$ & MIN OF $d=0$ \\
\hline AAI & & & 48.98 & 49.14 & 49.70 & 50.27 & 51.55 & 53.02 & 51.99 & 55.86 & 60.43 & 64.61 & 68.39 \\
\hline \multirow[t]{20}{*}{ P-NCC } & & & 91.09 & 90.24 & 88.66 & 86.49 & 78.16 & 66.09 & 54.78 & 48.66 & 47.03 & 45.91 & 45.15 \\
\hline & 0 & 1 & 91.09 & 90.24 & 88.66 & 86.49 & 78.16 & 66.09 & 54.78 & 48.66 & 47.03 & 45.91 & 45.15 \\
\hline & 0.1 & 0.9 & 86.88 & 86.13 & 84.77 & 82.87 & 75.50 & 64.78 & 54.50 & 49.38 & 48.37 & 47.78 & 47.47 \\
\hline & 0.15 & 0.85 & 84.77 & 84.08 & 82.82 & 81.06 & 74.17 & 64.13 & 54.36 & 49.74 & 49.04 & 48.71 & 48.63 \\
\hline & 0.2 & 0.8 & 82.67 & 82.02 & 80.87 & 79.25 & 72.84 & 63.47 & 54.22 & 50.10 & 49.71 & 49.65 & 49.80 \\
\hline & 0.25 & 0.75 & 80.56 & 79.97 & 78.92 & 77.43 & 71.51 & 62.82 & 54.08 & 50.46 & 50.38 & 50.58 & 50.96 \\
\hline & 0.3 & 0.7 & 78.46 & 77.91 & 76.97 & 75.62 & 70.18 & 62.17 & 53.94 & 50.82 & 51.05 & 51.52 & 52.12 \\
\hline & 0.35 & 0.65 & 76.35 & 75.86 & 75.02 & 73.81 & 68.85 & 61.51 & 53.80 & 51.18 & 51.72 & 52.45 & 53.28 \\
\hline & 0.4 & 0.6 & 74.24 & 73.80 & 73.08 & 72.00 & 67.51 & 60.86 & 53.66 & 51.54 & 52.39 & 53.39 & 54.45 \\
\hline & 0.45 & 0.55 & 72.14 & 71.75 & 71.13 & 70.19 & 66.18 & 60.21 & 53.52 & 51.90 & 53.06 & 54.32 & 55.61 \\
\hline & 0.5 & 0.5 & 70.03 & 69.69 & 69.18 & 68.38 & 64.85 & 59.55 & 53.38 & 52.26 & 53.73 & 55.26 & 56.77 \\
\hline & 0.55 & 0.45 & 67.93 & 67.63 & 67.23 & 66.57 & 63.52 & 58.90 & 53.24 & 52.62 & 54.40 & 56.19 & 57.93 \\
\hline & 0.6 & 0.4 & 65.82 & 65.58 & 65.28 & 64.76 & 62.19 & 58.25 & 53.10 & 52.98 & 55.07 & 57.13 & 59.09 \\
\hline & 0.65 & 0.35 & 63.72 & 63.52 & 63.33 & 62.95 & 60.86 & 57.59 & 52.96 & 53.34 & 55.74 & 58.06 & 60.26 \\
\hline & 0.7 & 0.3 & 61.61 & 61.47 & 61.39 & 61.13 & 59.53 & 56.94 & 52.82 & 53.70 & 56.41 & 59.00 & 61.42 \\
\hline & 0.75 & 0.25 & 59.50 & 59.41 & 59.44 & 59.32 & 58.20 & 56.28 & 52.69 & 54.06 & 57.08 & 59.93 & 62.58 \\
\hline & 0.8 & 0.2 & 57.40 & 57.36 & 57.49 & 57.51 & 56.87 & 55.63 & 52.55 & 54.42 & 57.75 & 60.87 & 63.74 \\
\hline & 0.85 & 0.15 & 55.29 & 55.30 & 55.54 & 55.70 & 55.54 & 54.98 & 52.41 & 54.78 & 58.42 & 61.80 & 64.90 \\
\hline & 0.9 & 0.1 & 53.19 & 53.25 & 53.59 & 53.89 & 54.21 & 54.32 & 52.27 & 55.14 & 59.09 & 62.74 & 66.07 \\
\hline & 1 & 0 & 48.98 & 49.14 & 49.70 & 50.27 & 51.55 & 53.02 & 51.99 & 55.86 & 60.43 & 64.61 & 68.39 \\
\hline
\end{tabular}

Table 17: Preference Model for N-P Ratio 3 Case

\section{N-P Ratio Changes from 6 to 9}

We shift N-P Ratio 6 patients per nurse per shift to 9 patients per nurse per shift with normal distribution with a mean of 0.50 (acuity scores fall in the range $[0 \ldots 1.0]$ ). 
The patient number will remain 180 while the number of nurses will decrease from 21 to 14 in order to reach one nurse cares 9 patients. Most nurses work consecutive 3 days (45.16\%) or 4 days $(45.16 \%)$ per week, fewer nurses $(9.68 \%)$ (due to the complexity of schedule, nurse work isolated shifts increased) work isolated days on or day off, each nurse will work 14 days per month which fit the real nurse scheduling.

The average total potential continuity of care relationship is 1033.28 among all 50 datasets. The actual usage of continuity of care relationship is 944.76 among all 50 datasets. We use the actual usage divided by the total potential to get the usage of continuity of care. The actual usage of continuity of care among 50 datasets is $91.43 \%$. The remaining unused continuity of care contained (8.57\%) two parts: 50.52 (4.89\%) unused continuity of care relationship (multiple nurses cared one patient before, we could only choose one care relationship and unselected other existed relationships); 38.00 (3.68\%) discarded continuity of care relationship (one nurse cared one patient before, but based on N-P Ratio, workload balance among all nurses, these care relationship could not be used, and these patients have to be assigned based on workload).

\begin{tabular}{lll}
\hline Method & AAI & P-NCC \\
\hline d=1.0 (WB) & 12.96 & $75.35 \%$ \\
d=0.9 & 13.05 & $74.50 \%$ \\
d=0.8 & 13.19 & $73.77 \%$ \\
d=0.7 & 13.29 & $72.52 \%$ \\
d=0.6 & 15.53 & $66.98 \%$ \\
d=0.5 & 20.86 & $58.08 \%$ \\
d=0.4 & 27.91 & $48.95 \%$ \\
d=0.3 & 33.17 & $43.55 \%$ \\
d=0.2 & 35.95 & $42.30 \%$ \\
d=0.1 & 39.67 & $41.33 \%$ \\
d=0 (CC-WB) & 42.33 & $40.65 \%$ \\
\hline
\end{tabular}

Table 18: AAI Vs P-NCC for N-P Ratio 9 
Based on Table 18, the whole trend of AAI vs P-NCC for N-P Ratio 9 case move to left compare to the baseline case, which means lower AAI range along with similar PNCC. We conduct to a numerical analysis of the solution based on a preference model in Table 19, which we provided weight 1 for the objective of minimizing AAI and weight 2 for the objective of minimizing P-NCC.

\begin{tabular}{|c|c|c|c|c|c|c|c|c|c|c|c|c|c|}
\hline & W1 for AAI & W2 for $\mathrm{P}-\mathrm{NCC}$ & MIN OF $d=1$ & MIN OF $d=0.9$ & MIN OF d $=0.8$ & MIN OF d $=0.7$ & MIN OF $d=0.6$ & MIN OF $d=0.5$ & MIN OF $d=0.4$ & MIN OF $d=0.3$ & MIN OF $d=0.2$ & MIN OF $d=0.1$ & MIN OF $d=0$ \\
\hline AAI & & & 12.96 & 13.05 & 13.19 & 13.29 & 15.53 & 20.86 & 27.91 & 33.17 & 35.95 & 39.67 & 42.33 \\
\hline \multirow[t]{20}{*}{ P-NCC } & & & 75.35 & 74.50 & 73.77 & 72.52 & 66.98 & 58.08 & 48.95 & 43.55 & 42.30 & 41.33 & 40.65 \\
\hline & 0 & 1 & 75.35 & 74.50 & 73.77 & 72.52 & 66.98 & 58.08 & 48.95 & 43.55 & 42.30 & 41.33 & 40.65 \\
\hline & 0.1 & 0.9 & 69.11 & 68.36 & 67.71 & 66.59 & 61.83 & 54.36 & 46.85 & 42.51 & 41.67 & 41.16 & 40.82 \\
\hline & 0.15 & 0.85 & 65.99 & 65.28 & 64.68 & 63.63 & 59.26 & 52.50 & 45.79 & 41.99 & 41.35 & 41.08 & 40.90 \\
\hline & 0.2 & 0.8 & 62.87 & 62.21 & 61.65 & 60.67 & 56.69 & 50.64 & 44.74 & 41.47 & 41.03 & 41.00 & 40.99 \\
\hline & 0.25 & 0.75 & 59.75 & 59.14 & 58.62 & 57.71 & 54.12 & 48.78 & 43.69 & 40.95 & 40.72 & 40.91 & 41.07 \\
\hline & 0.3 & 0.7 & 56.63 & 56.07 & 55.60 & 54.75 & 51.54 & 46.91 & 42.64 & 40.43 & 40.40 & 40.83 & 41.15 \\
\hline & 0.35 & 0.65 & 53.51 & 52.99 & 52.57 & 51.79 & 48.97 & 45.05 & 41.59 & 39.91 & 40.08 & 40.75 & 41.24 \\
\hline & 0.4 & 0.6 & 50.40 & 49.92 & 49.54 & 48.83 & 46.40 & 43.19 & 40.54 & 39.39 & 39.76 & 40.66 & 41.32 \\
\hline & 0.45 & 0.55 & 47.28 & 46.85 & 46.51 & 45.86 & 43.83 & 41.33 & 39.48 & 38.88 & 39.45 & 40.58 & 41.41 \\
\hline & 0.5 & 0.5 & 44.16 & 43.77 & 43.48 & 42.90 & 41.25 & 39.47 & 38.43 & 38.36 & 39.13 & 40.50 & 41.49 \\
\hline & 0.55 & 0.45 & 41.04 & 40.70 & 40.45 & 39.94 & 38.68 & 37.61 & 37.38 & 37.84 & 38.81 & 40.41 & 41.58 \\
\hline & 0.6 & 0.4 & 37.92 & 37.63 & 37.42 & 36.98 & 36.11 & 35.75 & 36.33 & 37.32 & 38.49 & 40.33 & 41.66 \\
\hline & 0.65 & 0.35 & 34.80 & 34.56 & 34.40 & 34.02 & 33.54 & 33.89 & 35.28 & 36.80 & 38.18 & 40.25 & 41.74 \\
\hline & 0.7 & 0.3 & 31.68 & 31.48 & 31.37 & 31.06 & 30.96 & 32.03 & 34.22 & 36.28 & 37.86 & 40.16 & 41.83 \\
\hline & 0.75 & 0.25 & 28.56 & 28.41 & 28.34 & 28.10 & 28.39 & 30.16 & 33.17 & 35.76 & 37.54 & 40.08 & 41.91 \\
\hline & 0.8 & 0.2 & 25.44 & 25.34 & 25.31 & 25.14 & 25.82 & 28.30 & 32.12 & 35.24 & 37.22 & 40.00 & 42.00 \\
\hline & 0.85 & 0.15 & 22.32 & 22.27 & 22.28 & 22.17 & 23.25 & 26.44 & 31.07 & 34.72 & 36.90 & 39.92 & 42.08 \\
\hline & 0.9 & 0.1 & 19.20 & 19.19 & 19.25 & 19.21 & 20.67 & 24.58 & 30.02 & 34.20 & 36.59 & 39.83 & 42.16 \\
\hline & 1 & 0 & 12.96 & 13.05 & 13.19 & 13.29 & 15.53 & 20.86 & 27.91 & 33.17 & 35.95 & 39.67 & 42.33 \\
\hline
\end{tabular}

Table 19: Preference Model for N-P Ratio 9 Case

Summary of Distribution of N-P Ratio changes

We report results where we shift N-P Ratio from six patients per nurse to three patients per nurse and nine patients per nurse. Figure 7 is a simplification of Table 17 and Table 19, in which we transfer the green points of the preference table into a curve. Based on the similar logic in Figure 4 [B], we show only the bottom half of the plot, up to P-NCC 0.5 in Figure 7.

Compared with the baseline case (six patients per nurse), the solution of N-P Ratio 3 has a significant increase in AAI due to the small number of patients per nurse. From $d=1.0$ to $d=0.5$ we get increments of PCC without a significant penalty of AAI, given that the initial AAI is high and the ability to decrease AAI is limited by the low 
number of patients per nurse. For N-P Ratio equal to 9 the solution curve is not significantly different from the baseline case, except the values for AAI are lower given that with a larger pool of patients per nurse we have greater opportunity to decrease work imbalance.

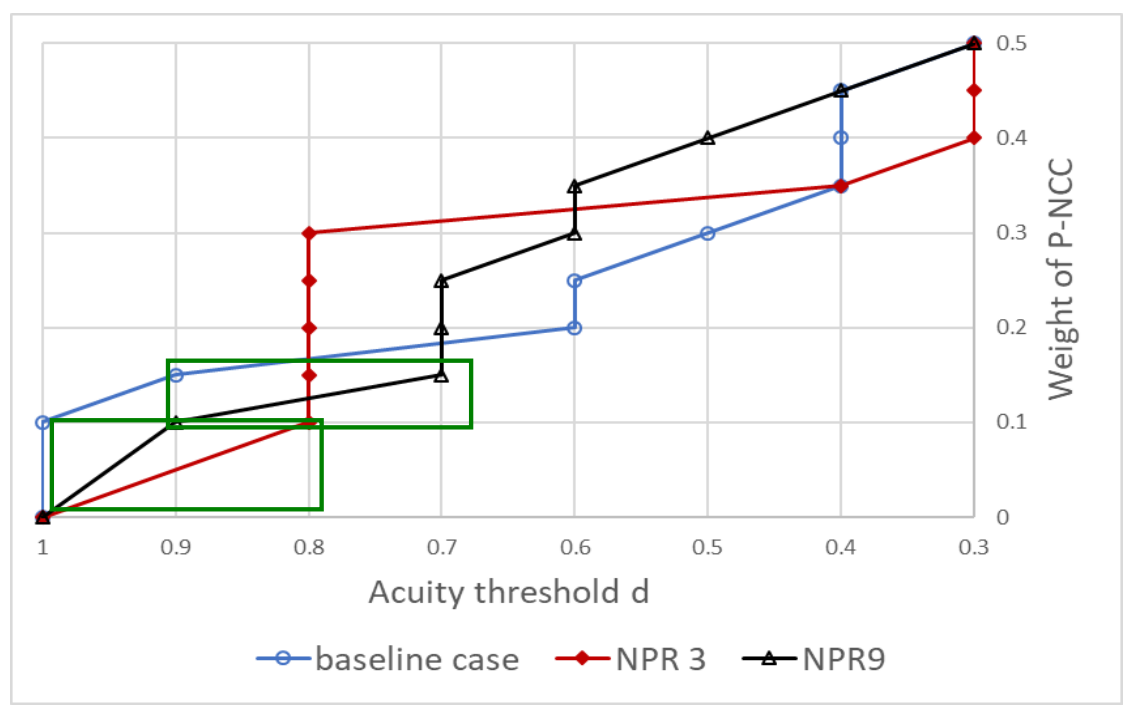

Figure 7: Example Horizonal Run of Case 3

In Figure 7, the N-P Ratio 3 curve on concentered from $d=1.0$ to $d=0.8$. In this case, one nurse will only care for three patients per shift, which is a little hard to achieve the workload balance. Therefore, our concentration range $(d=1.0$ to $d=0.8)$ is smaller compare to baseline case ( $d=0.9$ to $d=0.6$ ) since we consider more about workload imbalance.

Different from the N-P Ratio 3 curve, the N-P Ratio 9 curve on concentrated from $d=0.9$ to $d=0.7$. In this case, one nurse will care for nine patients per shift, which is a little easy to achieve the workload balance. Therefore, changes on the vertical axis $(0.1-0.15)$ are smaller compared to the baseline case (0.15-0.20). 


\section{Case 4: Patient with Special Skill Requirement}

Fourth, we assumed in the baseline case that all nurses are interchangeable (i.e., no special skills are required for any patients). In reality, patients have special needs such as foreign language, care activities such as intubation, or other special treatments, and need to be matched to a nurse with matching skill. In this scenario, we consider only one special need among the patient census. The percentage of patients with special care needs is set at $20 \%$. We set the percentage of nurses that have a matching skill at $40 \%$, to ensure that the number of patients with the special requirement will be less than the number of skilled nurses. There is no correlation between special skills requirements and patient acuity.

\begin{tabular}{lll}
\hline Method & AAI & P-NCC \\
\hline$d=1.0(W B)$ & 37.34 & $78.54 \%$ \\
d=0.9 & 36.51 & $77.89 \%$ \\
$d=0.8$ & 35.19 & $77.09 \%$ \\
$d=0.7$ & 35.25 & $75.65 \%$ \\
$d=0.6$ & 35.10 & $70.35 \%$ \\
$d=0.5$ & 37.49 & $62.03 \%$ \\
$d=0.4$ & 39.96 & $53.92 \%$ \\
$d=0.3$ & 43.30 & $49.27 \%$ \\
$d=0.2$ & 46.33 & $48.06 \%$ \\
$d=0.1$ & 49.25 & $47.14 \%$ \\
$d=0(C C-W B)$ & 51.95 & $46.60 \%$ \\
\hline
\end{tabular}

\section{Table 20: AAI Vs P-NCC for Case 4}

Based on Table 20, the whole trend of AAI vs P-NCC for special requirement case does change significantly at the start point (from 1.0- 0.5) compare to the baseline case. We conduct a numerical analysis of the solution based on a preference model in Table 21, which we provided weight 1 for the objective of minimizing AAI and weight 2 for the objective of minimizing P-NCC. 


\begin{tabular}{|c|c|c|c|c|c|c|c|c|c|c|c|c|c|}
\hline & W1 for AAI & W2 for P-NCC & MIN OF $d=1$ & MIN OF $d=0.9$ & MIN OF d $=0.8$ & MIN OF $d=0.7$ & MIN OF $d=0.6$ & MIN OF $d=0.5$ & MIN OF $d=0.4$ & MIN OF $d=0.3$ & MIN OF $d=0.2$ & MIN OF $d=0.1$ & MIN OF $d=0$ \\
\hline AAI & & & 37.34 & 36.51 & 35.19 & 35.25 & 35.10 & 37.49 & 39.96 & 43.30 & 46.33 & 49.25 & 51.95 \\
\hline \multirow[t]{20}{*}{ P-NCC } & & & 78.54 & 77.89 & 77.09 & 75.65 & 70.35 & 62.03 & 53.92 & 49.27 & 48.06 & 47.14 & 46.60 \\
\hline & 0 & 1 & 78.54 & 77.89 & 77.09 & 75.65 & 70.35 & 62.03 & 53.92 & 49.27 & 48.06 & 47.14 & 46.60 \\
\hline & 0.1 & 0.9 & 74.42 & 73.76 & 72.90 & 71.61 & 66.82 & 59.58 & 52.52 & 48.68 & 47.89 & 47.35 & 47.13 \\
\hline & 0.15 & 0.85 & 72.36 & 71.69 & 70.81 & 69.59 & 65.06 & 58.35 & 51.82 & 48.38 & 47.80 & 47.46 & 47.40 \\
\hline & 0.2 & 0.8 & 70.30 & 69.62 & 68.71 & 67.57 & 63.30 & 57.12 & 51.13 & 48.08 & 47.72 & 47.56 & 47.67 \\
\hline & 0.25 & 0.75 & 68.24 & 67.55 & 66.62 & 65.55 & 61.53 & 55.89 & 50.43 & 47.78 & 47.63 & 47.67 & 47.93 \\
\hline & 0.3 & 0.7 & 66.18 & 65.48 & 64.52 & 63.53 & 59.77 & 54.67 & 49.73 & 47.48 & 47.54 & 47.77 & 48.20 \\
\hline & 0.35 & 0.65 & 64.12 & 63.41 & 62.43 & 61.51 & 58.01 & 53.44 & 49.03 & 47.18 & 47.46 & 47.88 & 48.47 \\
\hline & 0.4 & 0.6 & 62.06 & 61.34 & 60.33 & 59.49 & 56.25 & 52.21 & 48.33 & 46.88 & 47.37 & 47.98 & 48.74 \\
\hline & 0.45 & 0.55 & 60.00 & 59.27 & 58.23 & 57.47 & 54.48 & 50.99 & 47.64 & 46.58 & 47.28 & 48.09 & 49.00 \\
\hline & 0.5 & 0.5 & 57.94 & 57.20 & 56.14 & 55.45 & 52.72 & 49.76 & 46.94 & 46.29 & 47.20 & 48.19 & 49.27 \\
\hline & 0.55 & 0.45 & 55.88 & 55.13 & 54.04 & 53.43 & 50.96 & 48.53 & 46.24 & 45.99 & 47.11 & 48.30 & 49.54 \\
\hline & 0.6 & 0.4 & 53.82 & 53.06 & 51.95 & 51.41 & 49.20 & 47.30 & 45.54 & 45.69 & 47.02 & 48.40 & 49.81 \\
\hline & 0.65 & 0.35 & 51.76 & 50.99 & 49.85 & 49.39 & 47.43 & 46.08 & 44.84 & 45.39 & 46.94 & 48.51 & 50.07 \\
\hline & 0.7 & 0.3 & 49.70 & 48.93 & 47.76 & 47.37 & 45.67 & 44.85 & 44.15 & 45.09 & 46.85 & 48.61 & 50.34 \\
\hline & 0.75 & 0.25 & 47.64 & 46.86 & 45.66 & 45.35 & 43.91 & 43.62 & 43.45 & 44.79 & 46.76 & 48.72 & 50.61 \\
\hline & 0.8 & 0.2 & 45.58 & 44.79 & 43.57 & 43.33 & 42.15 & 42.40 & 42.75 & 44.49 & 46.68 & 48.82 & 50.88 \\
\hline & 0.85 & 0.15 & 43.52 & 42.72 & 41.47 & 41.31 & 40.38 & 41.17 & 42.05 & 44.19 & 46.59 & 48.93 & 51.14 \\
\hline & 0.9 & 0.1 & 41.46 & 40.65 & 39.38 & 39.29 & 38.62 & 39.94 & 41.35 & 43.90 & 46.50 & 49.04 & 51.41 \\
\hline & 1 & 0 & 37.34 & 36.51 & 35.19 & 35.25 & 35.10 & 37.49 & 39.96 & 43.30 & 46.33 & 49.25 & 51.95 \\
\hline
\end{tabular}

Table 21: Preference Model for 20\% Special Requirement Case

Figure $\mathbf{8}$ is a simplification of Table 21, in which we transfer the green points of the preference table into a curve. Based on the similar logic in Figure 4 [B], we show only the bottom half of the plot, up to P-NCC 0.5 in Figure 8.

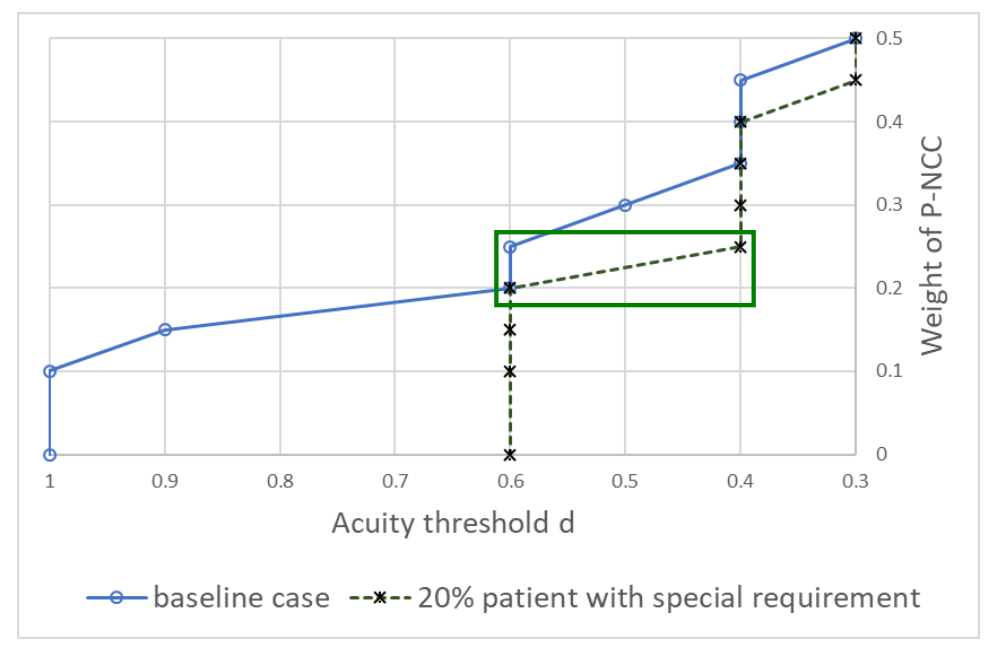

Figure 8: Example Horizontal Run of Case 4

The special requirement reduces the size of the pool for continuity and acuity-based assignment. In general AAI increases and the range of PCC becomes narrower when compared with the baseline case. There is an overlap between special skills requirement and continuity of care, as the pool of patients that needs special skills may still see the same 
nurse because of skill match, that sustains the PCC goal. There is an incentive to start at lower values of the acuity threshold $(d=0.6)$ for NPA purpose, $20 \%$ of patients are being assigned on special needs.

Based on Figure 8, 20\% special requirement case concentered from $d=0.6$ to $d$ $=0.4$. In this case, the $20 \%$ of patients will be assigned to nurses based on patients need match nurses' skill, along with continuity of care and workload balance approaches together to make the patient assignments hard to achieve the workload balance. Therefore, we would rather have more patients in the continuity of care group (impact point is 0.6 compared to 0.9 for the baseline case), and the threshold choices are concentered in a specific range.

\section{Case 5: Add Nurses from Pool if N-P Ratio exceed 6}

In the baseline case, we allow the number of patients per nurse to exceed this N-P Ratio given the stochastic nature of the simulation data, even though the N-P Ratio averaged across 56 shifts is 1:6. In hospital settings, the nurse manager would call in additional nursing staff from a pool to avoid exceeding the desired N-P Ratio. In this case, we consider the possibility of adding nurses from an out-of-unit pool in shifts where the number of patients needing care exceeds the desired N-P Ratio for the scheduled nursing staff (6 patients per nurse).

The following setting was used: pre-arrange nurses working schedule for 1 month (21 nurses will be separated into day cared group and night cared group) assigned by 3 shifts per week or 4 shifts per week; for every shift, if N-P Ratio > 6, add additional nurse from pool until N-P Ratio $<=6$. 
Based on Table 22, the results from this special case do not vary significantly when compared to the baseline case. We did not provide the table of preference here since the overall performance between baseline case and add nurse from pool case is not significantly different.

\begin{tabular}{|c|c|c|}
\hline Method & AAI & P-NCC \\
\hline $\mathrm{d}=1.0(\mathrm{WB})$ & 24.21 & $84.28 \%$ \\
\hline $\mathrm{d}=0.9$ & 24.31 & $83.54 \%$ \\
\hline $\mathrm{d}=0.8$ & 24.63 & $82.38 \%$ \\
\hline $\mathrm{d}=0.7$ & 24.94 & $80.63 \%$ \\
\hline $\mathrm{d}=0.6$ & 26.20 & $73.52 \%$ \\
\hline $\mathrm{d}=0.5$ & 30.05 & $62.49 \%$ \\
\hline $\mathrm{d}=0.4$ & 34.66 & $51.58 \%$ \\
\hline $\mathrm{d}=0.3$ & 40.60 & $45.29 \%$ \\
\hline $\mathrm{d}=0.2$ & 44.62 & $43.79 \%$ \\
\hline $\mathrm{d}=0.1$ & 50.07 & $42.65 \%$ \\
\hline $\mathrm{d}=0(\mathrm{CC}-\mathrm{WB})$ & 54.29 & $41.89 \%$ \\
\hline
\end{tabular}

Table 22: AAI Vs P-NCC for Case 5

We only provided Figure 9 here to show how to add a nurse from the pool case curve is similar to the baseline curve. Similar to the baseline case, the threshold choices of the concentrated choices of add nurse from pool case are shown from $d=0.9$ until $d=0.6$ for the weight of P-NCC $(0.15-0.20)$. Compare to the baseline case, the Case 5 add float nurse from the nurse pool into patients' assignments in a shift, which did not impact the assignment results for workload balance. Therefore, the results of Case 5 did not show significantly different compared to the baseline case. 


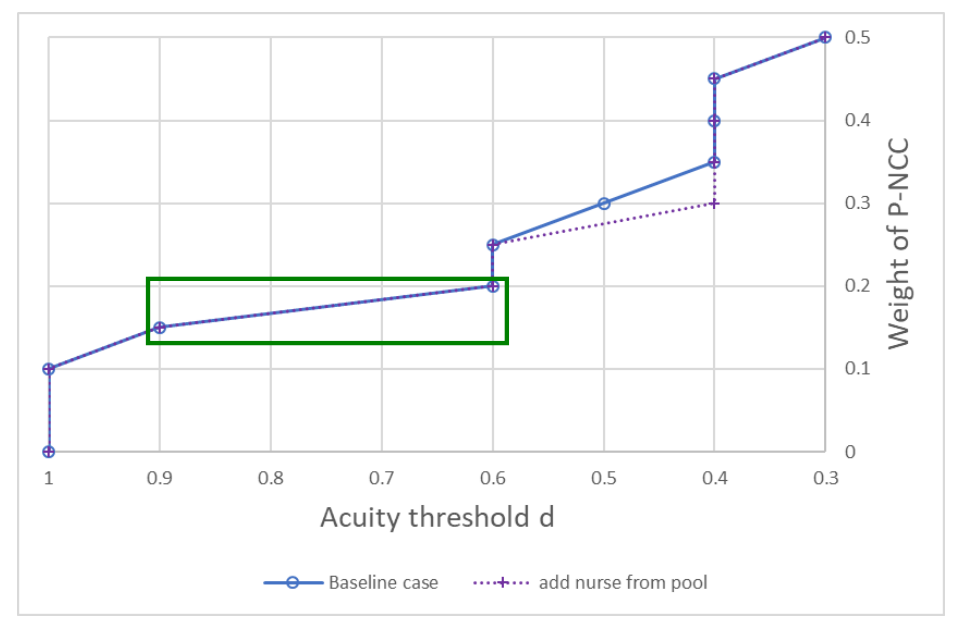

Figure 9: Example Horizontal Run of Case 5

Summary of Sensitivity Analysis

The analysis of the impact of several contextual factors supports the benefit of the proposed model with the segmentation of patients based on acuity to prioritize continuity of care versus workload balance NP assignments.

For the variation of shift length, the hard constraint on NP-ratio, N-P Ratio $=9$ and left-skewed distribution of acuity, we observe small variations of workload imbalance, increase in the first two cases and decrease in case of N-P Ratio $=9$ and left-skewed distribution. In settings where workload balance is more difficult either due to the distribution of acuity (right skewed), or NP assignment obeying other priority (special skills requirement), the acuity threshold tends to be concentrated on intermediate values, $d$ $=0.6$ to $d=0.3$ depending on preference weights, a similar result to the baseline model. There is a significant gain in PCC by moving from $\mathrm{d}=1$ to intermediate values relative to the increase in AAI. In settings with the low number of patients per nurse (N-P Ratio 1:3) or left skewed distribution, we see a concentration on either high or low values of the acuity 
threshold $d$, depending on the preference weight. Interestingly, the two cases differ in terms of potential for workload imbalance, being high in the case of N-P Ratio=3 and low in the case of left-skewed distribution.

Results for High Acuity Patients and Actual Usage of Continuity of Care One interesting finding from our model relates to the PCC obtained for patients with higher acuity levels in Table 23. For the purpose of illustration, we set the threshold for high acuity at $\mathrm{d}=0.6$ and tracked the PCC performance for this group of patients across the different models. Recall that the highest PCC obtained in the baseline model is $59.33 \%$ when $d=0$ (CC-WB method). Varying the acuity threshold, we obtain a maximum PCC for high acuity patients of $63.29 \%$. Continuity of care is arguably more important for critical-care patients than low acuity patients, so this focus would seem justified, delivering PCC improvement where it counts the most. This finding is consistent across the different cases considered in the sensitivity analysis.

\begin{tabular}{lllllllll}
\hline & Baseline & Left & Right & 8 hours & N-P & N-P & $20 \%$ & Add \\
Method & case & $15 \%$ & $15 \%$ & shift & Ratio 3 & Ratio 9 & patient & Nurse \\
\hline$d=1.0$ & $17.53 \%$ & $17.55 \%$ & $17.38 \%$ & $23.87 \%$ & $8.82 \%$ & $24.86 \%$ & $22.75 \%$ & $16.13 \%$ \\
$d=0.9$ & $20.49 \%$ & $17.63 \%$ & $20.03 \%$ & $27.01 \%$ & $13.02 \%$ & $27.66 \%$ & $25.31 \%$ & $19.19 \%$ \\
$d=0.8$ & $25.97 \%$ & $23.92 \%$ & $25.00 \%$ & $31.74 \%$ & $19.43 \%$ & $31.44 \%$ & $29.47 \%$ & $24.72 \%$ \\
$d=0.7$ & $33.25 \%$ & $39.04 \%$ & $39.48 \%$ & $38.63 \%$ & $28.18 \%$ & $37.20 \%$ & $35.26 \%$ & $32.06 \%$ \\
$d=0.6$ & $63.29 \%$ & $63.02 \%$ & $62.07 \%$ & $66.60 \%$ & $62.24 \%$ & $63.21 \%$ & $57.84 \%$ & $62.93 \%$ \\
$d=0.5$ & $62.99 \%$ & $62.10 \%$ & $60.60 \%$ & $65.44 \%$ & $60.69 \%$ & $62.73 \%$ & $57.12 \%$ & $62.08 \%$ \\
$d=0.4$ & $61.67 \%$ & $61.75 \%$ & $59.99 \%$ & $63.52 \%$ & $57.93 \%$ & $61.47 \%$ & $55.59 \%$ & $60.18 \%$ \\
$d=0.3$ & $60.17 \%$ & $60.28 \%$ & $59.69 \%$ & $61.51 \%$ & $55.93 \%$ & $60.15 \%$ & $54.19 \%$ & $59.00 \%$ \\
$d=0.2$ & $59.87 \%$ & $59.23 \%$ & $59.27 \%$ & $60.97 \%$ & $55.48 \%$ & $59.85 \%$ & $53.95 \%$ & $58.68 \%$ \\
$d=0.1$ & $59.45 \%$ & $58.46 \%$ & $59.27 \%$ & $61.12 \%$ & $54.97 \%$ & $59.49 \%$ & $53.69 \%$ & $58.29 \%$ \\
$d=0$ & $59.41 \%$ & $57.92 \%$ & $59.27 \%$ & $60.30 \%$ & $54.76 \%$ & $59.35 \%$ & $53.47 \%$ & $58.01 \%$ \\
\hline
\end{tabular}

Table 23: PCC Values for High Acuity Patient (d>0.6) 
The actual usage of potential care relationships is above $90 \%$ in 6 different conditions (baseline, left-skewed, right skewed, N-P Ratio 9, patient with the special need, and add additional nurses from the pool). Only 8 hours shift case and N-P Ratio 3 case showed different results $(72.45 \%$ for 8 hours shift case and $82.65 \%$ for N-P Ratio 3 case) of actual usage of potential continuity of care. For 8 hours shift case, we have lots of unused continuity of care relationship (multiple nurses cared one patient before, we could only choose one care relationship and unselected other existed relationships) (in this case, the nurse will have a much higher percentage to meet the same patients they used to care before, and there are lots of cases two or more nurses have care relationship with patients in current shift since patients remain 180 for 8 hours shift in 1 month, patients length of stay increased to range 6 shifts to 30 shifts distributed normally around a mean of 12.09 , nurse work $33 \%$ more shifts per week (from 3 shifts to 5 shifts per week), and total shifts changed from 54 to 81 (shifts changed from 12 hours to 8 hours. For the N-P Ratio 3 case, due to very limited N-P Ratio, we have to discard lots of care relationship, i.e., one nurse cared one patient before, but based on N-P Ratio, workload balance among all nurses, these care relationship could not be used (e.g., one nurse already has three patients with previous care relationship, and there is no more space for any assignment), and these patients have to be assigned based on workload. 
Chapter 6: Discussion, Conclusions, and Recommendations

NPA policies need to satisfy both objective goals such as workload distribution and productivity (Aickelin and Dowsland, 2004) and subjective goals (Waibel et al., 2011). Inspired by previous studies using quantitative parameters to distribute patients to nurses (Sadki et al., 2011, Schaus et al., 2009, Punnakitikashem et al., 2013) and studies demonstrating the benefits of familiarity between patients and nurses (McDonald et al., 2007, Austin et al., 1999), we developed a hybrid assignment algorithm which considers both quantitative goals, namely workload balance based on patient acuity (Latimer et al., 2009), and continuity of care relationship between nurses and patients from prior shifts (Allen, 2015). The method, termed CC-WB, gives priority to relationship-based assignments followed by workload balance.

We compared the CC-WB method to three other control cases: (1) the WB approach, which attempts to minimize acuity imbalance in workload assignments; (2) an approach we call $\mathrm{CC}$, where we consider only the goal of continuity of care (patients not assigned based on prior relationships are assigned randomly); and (3) the NB approach, which produces assignments based on solely numeric balance. In a baseline experiment, we tested all these four approaches in our simulated model for 56 shifts (4 weeks) and measured AAI, which captures the acuity imbalance produced by each method, and PCC, which measures the extent to which each method produces assignments with prior-care relationships.

The results of this experiment showed limitations on the continuity-based assignment imposed by the hospital work setting. The maximum overall PCC that could 
be obtained was $59.33 \%$ (baseline model) for the CC-WB model. The limitation to work in contiguous shifts and the number of patients per nurse limit the usage of prior-care relationships. Moreover, the results demonstrate the tradeoff space between relationship and workload imbalance. The WB method generates better acuity balance, the CC and CCWB approaches produced the greatest gains in terms of continuity of care. More interestingly, the CC-WB method provided the best balance between the goals, with a 47.66 percentage-point increase in PCC, in exchange for a $15.8 \%$ increase in AAI when compared with WB.

To address the tradeoff between acuity imbalance and relationship-based assignment, we relax the acuity-minimization and relationship-maximization goals, and we performed a sensitivity experiment in which we tested the CC-WB method for values of $d$ ranging from 0 to 1 . Here, changing the value of $d$ allowed us to use relationship-based assignment with a more precise application, such that we consider only higher-acuity patients (with acuity scores greater than or equal to $d$ ) for relationship-based assignment. The results of our experiments showed that mid-range values of $d$, where $d=0.5$ or $d=$ 0.6 , provided AAI results similar to the $\mathrm{WB}$ case (a maximum of $1.02 \%$ greater than WB for the $d=0.6$ case), while also providing PCC gains of 36.6 and 25.8 percentage points, respectively, in comparison to the WB case. Here, we effectively gain the benefits of relationship-based assignment for free, with little or no penalty in terms of acuity balance.

This study makes several contributions to research and practice. First, we consider a new problem in NPA related to assuring continuity of care to patients. We extend prior research on NPA by incorporating qualitative factors, recognizing that nursing work 
contains both objective work and subjective work, where subjective work requires addressing unpredictable factors (Weston et al., 2006, Griffin et al., 2007). Increasing continuity of care has a dual impact as not only reduces subjective workload for nurses but can also improve quality of care delivery to patients (Bodenheimer, 2008).

Secondly, we develop a stylized simulation model based on a survey of the literature, to test the effectiveness of different approaches to NPA. Discrete event simulation is well suited to the complexities of the hospital environment (Gnanlet and Gilland, 2009) and the NPA problem in particular. Using a simulated setting we were able to consider accumulated workload balance over four weeks while most prior studies optimize for work balance in the short term.

We used our simulation model to generate a broader understanding of the tradeoff between workload balance based on objective work and continuity of care. The relationship-based goal may impact the quality of care delivery and patient satisfaction, but also the perception of the subjective workload from nurses. A key insight is that by enabling a pool of patients with low acuity to be assigned to balance workload, we can achieve high levels of continuity of care without penalty on work balance

The methods presented in this article are useful for different stakeholders involved in nursing care in inpatient settings. Inspired by the assignment logic and important factors of NPA for charge nurses (Plover, 2017), this method can be embedded in a DSS tool and used to develop operational plans for NPA on a daily basis, facilitating administrative work. Hospital managers can break the tradeoff between objective workload balance and continuity of care, while maintaining high acuity patients under relationship-based 
treatment. The DSS model data can also be used to inform nurses' perception of workload balance in the long term. Finally, we note that, even though the relationship-based assignment could benefit both nurses and patients, a full relationship-based assignment is difficult to achieve in operational settings (Clarke and Donaldson, 2008). Our CC-WB method with acuity threshold takes advantage of this situation, by assigning based on prior care where possible, and based on acuity where a relationship-based assignment is not possible.

There are some limitations to this work. Our method did not consider patient admit or discharge during a shift. We used acuity as a major parameter to assign patients to nurses under workload balance; however, other quantitative factors could be substituted for acuity with little no change. Also, our patients' acuities are generated from normal distributions, which ignore that higher acuity also tends to have higher length of stay.

Our findings point to several opportunities for future work. We could consider inheriting prior positive care relationships between patients and nurses, and assign patients with negative care relationships based on acuity. Aside from acuity, we could assign patients based on their room location, diagnosis, or special needs. Finally, continuity of care might not be the only way to gain the benefits of qualitative goals. Other qualitative factors, such as patients' mental conditions, the complexity of patients' communication, or the stress level of nurses could also be used in nurse-patient assignment decision-making.

\section{Conclusion}

We proposed two hybrid assignment methods, i.e., CC-WB method and acuity threshold method, to address the need to provide continuity of care while ensuring fair workload 
distribution among nurses. The CC-WB method alleviates the tradeoff inherent to single criteria methods namely $\mathrm{CC}$ and WB. Based on the first-round simulation results, we further proposed a multi-criteria balancing method with acuity threshold. Varying the acuity threshold for prioritizing continuity-based assignments enables higher continuity with a limited impact on workload balance. Maintaining a pool of patients with lower acuity to assign based on workload balance enables decision makers to break the tradeoff between the continuity of care and the workload balance. 


\section{REFERENCES}

ABDELGHANY, K. F., ABDELGHANY, A. F. \& EKOLLU, G. 2008. An integrated decision support tool for airlines schedule recovery during irregular operations. European Journal of Operational Research, 185, 825-848.

AICKELIN, U. \& DOWSLAND, K. A. 2004. An indirect genetic algorithm for a nursescheduling problem. Computers \& Operations Research, 31, 761-778.

AIKEN, L. H., SLOANE, D. M., BRUYNEEL, L., VAN DEN HEEDE, K., GRIFFITHS, P., BUSSE, R., DIOMIDOUS, M., KINNUNEN, J., KÓZKA, M. \& LESAFFRE, E. 2014. Nurse staffing and education and hospital mortality in nine European countries: a retrospective observational study. The Lancet, 383, 1824-1830.

ALGHAMDI, M. G. 2016. Nursing workload: a concept analysis. Journal of nursing management, 24, 449-457.

ALLEN, S. B. 2015. The Nurse-Patient Assignment: Purposes and Decision Factors. $J$ Nurs Adm, 45, 628-35.

AMOAKO-GYAMPAH, K. \& MEREDITH, J. R. 1996. A simulation study of FMS tool allocation procedures. Journal of manufacturing systems, 15, 419-431.

AUERBACH, D. I., STAIGER, D. O., MUENCH, U. \& BUERHAUS, P. I. 2013. The nursing workforce in an era of health care reform. New England Journal of Medicine, 368, 1470-1472.

AUSTIN, D. J., BONTEN, M. J. M., WEINSTEIN, R. A., SLAUGHTER, S. \& ANDERSON, R. M. Vancomycin-resistant enterococci in intensive-care hospital settings: Transmission dynamics, persistence, and the impact of infection control programs. Proceedings of the National Academy of Sciences, June 8, 1999. 69086913.

AZAIEZ, M. N. \& AL SHARIF, S. S. 2005. A 0-1 goal programming model for nurse scheduling. Computers \& Operations Research, 32, 491-507. 
BAKER, R. L., TINDELL, S., BUCKLEY BEHAN, D., TURPIN, P. G., ROSENBERGER, J. M. \& PUNNAKITIKASHEM, P. 2010. Phase I: Creating an Electronic Prototype to Generate Equitable Hospital Nurse-to-Patient Assignments. CIN: Computers, Informatics, Nursing, 28, 57-62.

BODENHEIMER, T. 2008. Coordinating care-a perilous journey through the health care system. Mass Medical Soc.

BOWERS, J., CHEYNE, H., MOULD, G. \& PAGE, M. 2015. Continuity of care in community midwifery. Health care management science, 18, 195-204.

BRENNAN, C. W. \& DALY, B. J. 2009. Patient acuity: A concept analysis. Journal of advanced nursing, 65, 1114-1126.

BRODOVICZ, K. G., MEHTA, V., ZHANG, Q., ZHAO, C., DAVIES, M. J., CHEN, J., RADICAN, L. \& ENGEL, S. S. 2013. Association between hypoglycemia and inpatient mortality and length of hospital stay in hospitalized, insulin-treated patients. Curr Med Res Opin, 29, 101-7.

BRUNT, B. A. 2005. Critical thinking in nursing: An integrated review. The Journal of Continuing Education in Nursing, 36, 60-67.

BUERHAUS, P. I., DONELAN, K., ULRICH, B. T. \& NORMAN, L. 2005. Hospital RNs' and CNOs' perceptions of the impact of the nursing shortage on the quality of care. Nursing Economics, 23, 214.

BURKE, E. K., DE CAUSMAECKER, P., BERGHE, G. V. \& VAN LANDEGHEM, H. 2004. The state of the art of nurse rostering. Journal of scheduling, 7, 441-499.

BURKE, E. K., DE CAUSMAECKER, P., DE MAERE, G., MULDER, J., PAELINCK, M. \& BERGHE, G. V. 2010. A multi-objective approach for robust airline scheduling. Computers \& Operations Research, 37, 822-832. 
BUSHNELL, P. T., COLOMBI, A., CARUSO, C. C. \& TAK, S. 2010. Work schedules and health behavior outcomes at a large manufacturer. Ind Health, 48, 395-405.

CARUSO, C. C. 2014. Negative Impacts of Shiftwork and Long Work Hours. Rehabilitation nursing : the official journal of the Association of Rehabilitation Nurses, 39, 16-25.

CERTA, A., ENEA, M., GALANTE, G. \& MANUELA LA FATA, C. 2009. Multiobjective human resources allocation in $\mathrm{R} \& \mathrm{D}$ projects planning. International Journal of Production Research, 47, 3503-3523.

CLARKE, S. P. \& DONALDSON, N. E. 2008. Chapter 25. Nurse staffing and patient care quality and safety. Patient safety and quality: an evidence-based handbook for nurses. Rockville, MD: Agency for Healthcare Research and Quality.

CLEARY, P. D., GREENFIELD, S., MULLEY, A. G., PAUKER, S. G., SCHROEDER, S. A., WEXLER, L. \& MCNEIL, B. J. 1991. Variations in length of stay and outcomes for six medical and surgical conditions in Massachusetts and California. Jama, 266, 73-79.

COHN, A., ROOT, S., KYMISSIS, C., ESSES, J. \& WESTMORELAND, N. 2009. Scheduling medical residents at boston university school of medicine. Interfaces, 39, 186-195.

CORDERY, J. L., MORRISON, D., WRIGHT, B. M. \& WALL, T. D. 2010. The impact of autonomy and task uncertainty on team performance: A longitudinal field study. Journal of Organizational Behavior, 31, 240-258.

DENTON, B. T. 2013. Handbook of healthcare operations management, Springer.

DONG, S., JOHAR, M. S. \& KUMAR, R. L. 2011. A benchmarking model for management of knowledge-intensive service delivery networks. Journal of Management Information Systems, 28, 127-160.

DUFFIELD, C., DIERS, D., O'BRIEN-PALLAS, L., AISBETT, C., ROCHE, M., KING, M. \& AISBETT, K. 2011. Nursing staffing, nursing workload, the work environment and patient outcomes. Applied nursing research, 24, 244-255. 
ESPINOSA, J. A., SLAUGHTER, S. A., KRAUT, R. E. \& HERBSLEB, J. D. 2007. Familiarity, complexity, and team performance in geographically distributed software development. Organization science, 18, 613-630.

FERRAND, Y. B., MAGAZINE, M. J. \& RAO, U. S. 2014. Partially flexible operating rooms for elective and emergency surgeries. Decision Sciences, 45, 819-847.

FIKAR, C. \& HIRSCH, P. 2017. Home health care routing and scheduling: A review. Computers \& Operations Research, 77, 86-95.

FREI, F. X. 2006. Breaking the tradeoff between efficiency and service. Harvard business review, 84, 92.

FÜGENER, A., BRUNNER, J. O. \& PODTSCHASKE, A. 2015. Duty and workstation rostering considering preferences and fairness: a case study at a department of anaesthesiology. International Journal of Production Research, 53, 7465-7487.

GANGULY, S., LAWRENCE, S. \& PRATHER, M. 2014. Emergency department staff planning to improve patient care and reduce costs. Decision Sciences, 45, 115-145.

GARRETT, D. K. \& MCDANIEL, A. M. 2001. A new look at nurse burnout: the effects of environmental uncertainty and social climate. Journal of Nursing Administration, 31, 91-96.

GNANLET, A. \& GILLAND, W. G. 2009. Sequential and simultaneous decision making for optimizing health care resource flexibilities. Decision Sciences, 40, 295-326.

GOCGUN, Y. \& PUTERMAN, M. L. 2014. Dynamic scheduling with due dates and time windows: an application to chemotherapy patient appointment booking. Health care management science, 17, 60-76.

GONCALVES, L. A., PADILHA, K. G. \& CARDOSO SOUSA, R. M. 2007. Nursing activities score (NAS): a proposal for practical application in intensive care units. Intensive Crit Care Nurs, 23, 355-61. 
GOODMAN, P. S. \& LEYDEN, D. P. 1991. Familiarity and group productivity. Journal of Applied Psychology, 76, 578.

GRIFFIN, M. A., NEAL, A. \& PARKER, S. K. 2007. A new model of work role performance: Positive behavior in uncertain and interdependent contexts. Academy of management journal, 50, 327-347.

HAGGERTY, J. L., REID, R. J., FREEMAN, G. K., STARFIELD, B. H., ADAIR, C. E. \& MCKENDRY, R. 2003. Continuity of care: a multidisciplinary review. BMJ: British Medical Journal, 327, 1219.

HARPER, K. \& MCCULLY, C. 2007. Acuity Systems Dialogue and Patient Classification System Essentials. Nursing Administration Quarterly, 31, 284-299.

HARRISON, D. A., MOHAMMED, S., MCGRATH, J. E., FLOREY, A. T. \& VANDERSTOEP, S. W. 2003. Time matters in team performance: Effects of member familiarity, entrainment, and task discontinuity on speed and quality. Personnel Psychology, 56, 633-669.

HILLIER, M. 2013. Designing unpaced production lines to optimize throughput and workin-process inventory. IIE Transactions, 45, 516-527.

HORNUNG, T., KOSCHMIDER, A. \& LAUSEN, G. Recommendation based process modeling support: Method and user experience. International Conference on Conceptual Modeling, 2008. Springer, 265-278.

KELLOGG, D. L. \& WALCZAK, S. 2007. Nurse scheduling: from academia to implementation or not? Interfaces, 37, 355-369.

KITTUR, A., NICKERSON, J. V., BERNSTEIN, M., GERBER, E., SHAW, A., ZIMMERMAN, J., LEASE, M. \& HORTON, J. The future of crowd work. Proceedings of the 2013 conference on Computer supported cooperative work, 2013. ACM, 1301-1318.

LAGANGA, L. R. \& LAWRENCE, S. R. 2007. Clinic overbooking to improve patient access and increase provider productivity. Decision Sciences, 38, 251-276. 
LANG, T. A., HODGE, M., OLSON, V., ROMANO, P. S. \& KRAVITZ, R. L. 2004. Nurse-patient ratios: a systematic review on the effects of nurse staffing on patient, nurse employee, and hospital outcomes. J Nurs Adm, 34, 326-37.

LANZARONE, E. \& MATTA, A. 2014. Robust nurse-to-patient assignment in home care services to minimize overtimes under continuity of care. Operations Research for Health Care, 3, 48-58.

LATIMER, M. A., JOHNSTON, C. C., RITCHIE, J. A., CLARKE, S. P. \& GILIN, D. 2009. Factors affecting delivery of evidence - based procedural pain care in hospitalized neonates. Journal of Obstetric, Gynecologic, \& Neonatal Nursing, 38, 182-194.

LEE, L. H., CHEW, E. P., TENG, S. \& CHEN, Y. 2008. Multi-objective simulation-based evolutionary algorithm for an aircraft spare parts allocation problem. European Journal of Operational Research, 189, 476-491.

LEE, L. H., LEE, C. U. \& TAN, Y. P. 2007. A multi-objective genetic algorithm for robust flight scheduling using simulation. European Journal of Operational Research, 177, 1948-1968.

LEITER, M. P., HARVIE, P. \& FRIZZELL, C. 1998. The correspondence of patient satisfaction and nurse burnout. Soc Sci Med, 47, 1611-7.

LIANG, B. \& TURKCAN, A. 2015. Acuity-based nurse assignment and patient scheduling in oncology clinics. Health Care Manag Sci.

LIM, K. H., BENBASAT, I. \& TODD, P. A. 1996. An experimental investigation of the interactive effects of interface style, instructions, and task familiarity on user performance. ACM Transactions on Computer-Human Interaction (TOCHI), 3, 137. 
LIU, M., YANG, D., SU, Q. \& XU, L. 2018. Bi-objective approaches for home healthcare medical team planning and scheduling problem. Computational and Applied Mathematics, 37, 4443-4474.

LOUCKS, J. S. \& JACOBS, F. R. 1991. Tour scheduling and task assignment of a heterogeneous work force: A heuristic approach. Decision Sciences, 22, 719-738.

MARTSOLF, G. R., AUERBACH, D., BENEVENT, R., STOCKS, C., JIANG, H. J., PEARSON, M. L., EHRLICH, E. D. \& GIBSON, T. B. 2014. Examining the value of inpatient nurse staffing: an assessment of quality and patient care costs. Medical care, 52, 982-988.

MASSEY, D., CHABOYER, W. \& ANDERSON, V. 2017. What factors influence ward nurses' recognition of and response to patient deterioration? An integrative review of the literature. Nursing open, 4, 6-23.

MCDONALD, K. M., SUNDARAM, V., BRAVATA, D. M., LEWIS, R., LIN, N., KRAFT, S. A., MCKINNON, M., PAGUNTALAN, H. \& OWENS, D. K. 2007. Closing the Quality Gap: A Critical Analysis of Quality Improvement Strategies (Vol. 7: Care Coordination), Rockville MD.

MCINTOSH, N., BURGESS JR, J. F., METERKO, M., RESTUCCIA, J. D., ALTWHITE, A. C., KABOLI, P. \& CHARNS, M. 2014. Impact of provider coordination on nurse and physician perceptions of patient care quality. Journal of nursing care quality, 29, 269-279.

MULlinAX, C. \& LAWLEY, M. 2002. Assigning Patients to Nurses in Neonatal Intensive Care. The Journal of the Operational Research Society, 53, 25-35.

MYNY, D., VAN HECKE, A., DE BACQUER, D., VERHAEGHE, S., GOBERT, M., DEFLOOR, T. \& VAN GOUBERGEN, D. 2012. Determining a set of measurable and relevant factors affecting nursing workload in the acute care hospital setting: a cross-sectional study. Int J Nurs Stud, 49, 427-36.

NEEDLEMAN, J., BUERHAUS, P., PANKRATZ, V. S., LEIBSON, C. L., STEVENS, S. R. \& HARRIS, M. 2011. Nurse staffing and inpatient hospital mortality. New England Journal of Medicine, 364, 1037-1045. 
PAKZAD, H., THEVENDRAN, G., PENNER, M. J., QIAN, H. \& YOUNGER, A. 2014. Factors associated with longer length of hospital stay after primary elective ankle surgery for end-stage ankle arthritis. J Bone Joint Surg Am, 96, 32-9.

PLOVER, C. 2017. An Exploration of Charge Nurse Decision-Making Related to the Nurse-Patient Assignment on Adult Medical-Surgical Inpatient Units, University of Pennsylvania.

PUNNAKITIKASHEM, P., ROSENBERBER, J. M. \& BUCKLEY-BEHAN, D. F. 2013. A stochastic programming approach for integrated nurse staffing and assignment. IIE Transactions, 45, 1059-1076.

PUNNAKITIKASHEM, P., ROSENBERGER, J. M., BUCKLEY-BEHAN, D. F., BAKER, R. L. \& GOSS, K. An optimization-based prototype for nurse assignment. the 7th Asian Pacific industrial engineering and management systems conference, 2006. 17-20.

PUNNAKITIKASHEM, P., ROSENBERGER, J. M. \& BUCKLEY BEHAN, D. 2008. Stochastic programming for nurse assignment. Computational Optimization and Applications, 40, 321-349.

REIJERS, H. A., JANSEN-VULLERS, M. H., ZUR MUEHLEN, M. \& APPL, W. Workflow management systems + swarm intelligence $=$ dynamic task assignment for emergency management applications. International Conference on Business Process Management, 2007. Springer, 125-140.

ROGOWSKI, J. A., STAIGER, D., PATRICK, T., HORBAR, J., KENNY, M. \& LAKE, E. T. 2013. Nurse staffing and NICU infection rates. JAMA pediatrics, 167, 444450.

SADKI, A., XIE, X. \& CHAUVIN, F. Appointment scheduling of oncology outpatients. Automation Science and Engineering (CASE), 2011 IEEE Conference on, 2011. IEEE, 513-518.

SANTIBANEZ, P., ARISTIZABAL, R., PUTERMAN, M. L., CHOW, V. S., HUANG, W., KOLLMANNSBERGER, C., NORDIN, T., RUNZER, N. \& TYLDESLEY, 
S. 2012. Operations research methods improve chemotherapy patient appointment scheduling. Jt Comm J Qual Patient Saf, 38, 541-53.

SCHAUS, P., VAN HENTENRYCK, P. \& RÉGIN, J.-C. 2009. Scalable Load Balancing in Nurse to Patient Assignment Problems. In: VAN HOEVE, W.-J. \& HOOKER, J. N. (eds.) Integration of AI and OR Techniques in Constraint Programming for Combinatorial Optimization Problems: 6th International Conference, CPAIOR 2009 Pittsburgh, PA, USA, May 27-31, 2009 Proceedings. Berlin, Heidelberg: Springer Berlin Heidelberg.

SCHOEN, C., OSBORN, R., HOW, S. K., DOTY, M. M. \& PEUGH, J. 2009. In chronic condition: experiences of patients with complex health care needs, in eight countries, 2008. Health affairs, 28, w1-w16.

SCOTT, L. D., ROGERS, A. E., HWANG, W. T. \& ZHANG, Y. 2006. Effects of critical care nurses' work hours on vigilance and patients' safety. Am J Crit Care, 15, 30-7.

SEVINC, S., SANLI, U. A. \& GOKER, E. 2013. Algorithms for scheduling of chemotherapy plans. Computers in biology and medicine, 43, 2103-2109.

SIMPSON, R. \& COURTNEY, R. 2002. Critical thinking in nursing education: Literature review. International journal of nursing practice, 8, 89-98.

SIR, M. Y., DUNDAR, B., BARKER STEEGE, L. M. \& PASUPATHY, K. S. 2015. Nurse-patient assignment models considering patient acuity metrics and nurses' perceived workload. Journal of Biomedical Informatics, 55, 237-248.

SPENCE, K., TARNOW-MORDI, W., DUNCAN, G., JAYASURYIA, N., ELLIOTT, J., KING, J. \& KITE, F. 2006. Measuring nursing workload in neonatal intensive care. Journal of nursing management, 14, 227-234.

STANTON, M. W. \& STANTON, M. W. 2004. Hospital nurse staffing and quality of care, Agency for Healthcare Research and Quality Rockville, MD. 
STIMPFEL, A. W., SLOANE, D. M. \& AIKEN, L. H. 2012. The longer the shifts for hospital nurses, the higher the levels of burnout and patient dissatisfaction. Health Aff (Millwood), 31, 2501-9.

SUN, J., LYSECKY, R., SHANKAR, K., KODI, A., LOURI, A. \& ROVEDA, J. 2014. Workload assignment considering NBTI degradation in multicore systems. ACM Journal on Emerging Technologies in Computing Systems (JETC), 10, 4.

SUNDARAMOORTHI, D., CHEN, V. C. P., ROSENBERGER, J. M., KIM, S. B. \& BUCKLEY-BEHAN, D. F. 2009. A data-integrated simulation model to evaluate nurse-patient assignments. Health Care Management Science, 12, 252-268.

VAN DEN BERGH, J., BELIËN, J., DE BRUECKER, P., DEMEULEMEESTER, E. \& DE BOECK, L. 2013. Personnel scheduling: A literature review. European Journal of Operational Research, 226, 367-385.

VAN SLYCK, A. \& JOHNSON, K. R. 2000. Using patient acuity data to manage patient care outcomes and patient care costs. Outcomes management for nursing practice, $5,36-40$.

VILE, J. L., GILLARD, J. W., HARPER, P. R. \& KNIGHT, V. A. 2017. A Queueing Theoretic Approach to Set Staffing Levels in Time - Dependent Dual - Class Service Systems. Decision Sciences, 48, 766-794.

WAIBEL, S., HENAO, D., ALLER, M.-B., VARGAS, I. \& VÁZQUEZ, M.-L. 2011. What do we know about patients' perceptions of continuity of care? A meta-synthesis of qualitative studies. International Journal for Quality in Health Care, 24, 39-48.

WESTON, M., BIRD, S., HELSEN, W., NEVILL, A. \& CASTAGNA, C. 2006. The effect of match standard and referee experience on the objective and subjective match workload of English Premier League referees. Journal of Science and Medicine in Sport, 9, 256-262.

WRIGHT, P. D., BRETTHAUER, K. M. \& CÔTÉ, M. J. 2006. Reexamining the nurse scheduling problem: Staffing ratios and nursing shortages. Decision Sciences, 37, 39-70. 
XIANG, W. 2017. A multi-objective ACO for operating room scheduling optimization. Natural Computing, 16, 607-617.

YANKOVIC, N. \& GREEN, L. V. 2011. Identifying good nursing levels: A queuing approach. Operations research, 59, 942-955.

ZUR MUEHLEN, M. 2004. Organizational management in workflow applications-issues and perspectives. Information Technology and Management, 5, 271-291. 
VITA

\section{HAOQIANG JIANG}

2014-2018

Ph.D., Business

Information Systems and Business Analytics

College of Business - Florida International

University

Miami, FL

2012-2014

Master of Business Administration concentrate on

Finance

Ball State University,

Muncie, IN

2009-2011

Master of Art in Sports Administration

Ball State University,

Muncie, IN

2004-2006

Bachelor of Art in Chinese Literature

Shanghai Normal University,

Shanghai, PR China

\section{PUBLICATIONS AND PRESENTATIONS}

Chen, C., \& H. Jiang (2018); "Important Skills for Data Scientists in China: Two Delphi Studies" accepted for publication in the Journal of Computer Information Systems, DOI. $10.1080 / 08874417.2018 .1472047$

H. Jiang, D. VanderMeer, \& P.Gomes (2018); "Balancing Workload and Care Communication Cost in Nurse Patient Assignment." Emergent Research Forum, AMCIS 2018, New Orleans, LA.

H.Subramanian, M.Chen \& H. Jiang (2017); "Entrepreneurship in BRIC: Who will venture capitalists fund?" AMCIS 2017, Boston, MA.

H. Jiang, J Li, \& D. VanderMeer; "Exploring the workload balance effects of including continuity-based factors in nurse-patient assignments", DESRIST 2016 Conference. St. John's, Canada. 